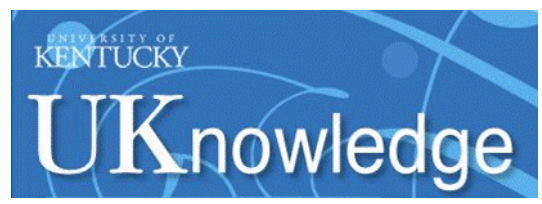

University of Kentucky

UKnowledge

$12-21-2015$

\title{
SDSS-IV/MaNGA: Spectrophotometric Calibration Technique
}

\author{
Renbin Yan \\ University of Kentucky, yanrenbin@uky.edu \\ Christy Tremonti \\ University of Wisconsin - Madison \\ Matthew A. Bershady \\ University of Wisconsin - Madison \\ David R. Law \\ Space Telescope Science Institute \\ David J. Schlegel \\ Lawrence Berkeley National Laboratory
}

See next page for additional authors

Follow this and additional works at: https://uknowledge.uky.edu/physastron_facpub

Part of the Astrophysics and Astronomy Commons, and the Physics Commons

Right click to open a feedback form in a new tab to let us know how this document benefits you.

\section{Repository Citation}

Yan, Renbin; Tremonti, Christy; Bershady, Matthew A.; Law, David R.; Schlegel, David J.; Bundy, Kevin; Drory, Niv; MacDonald, Nicholas; Bizyaev, Dmitry; Blanc, Guillermo A.; Blanton, Michael R.; Cherinka, Brian; Eigenbrot, Arthur; Gunn, James E.; Harding, Paul; Hogg, David W.; Sánchez-Gallego, José R.; Sánchez, Sebastian F.; Wake, David A.; Weijmans, Anne-Marie; Xiao, Ting; and Zhang, Kai, "SDSS-IV/MaNGA: Spectrophotometric Calibration Technique" (2015). Physics and Astronomy Faculty Publications. 423. https://uknowledge.uky.edu/physastron_facpub/423

This Article is brought to you for free and open access by the Physics and Astronomy at UKnowledge. It has been accepted for inclusion in Physics and Astronomy Faculty Publications by an authorized administrator of UKnowledge. For more information, please contact UKnowledge@lsv.uky.edu. 


\section{SDSS-IV/MaNGA: Spectrophotometric Calibration Technique}

\section{Digital Object Identifier (DOI)}

https://doi.org/10.3847/0004-6256/151/1/8

\section{Notes/Citation Information}

Published in The Astronomical Journal, v. 151, no. 1, 8, p. 1-18.

(c) 2016. The American Astronomical Society. All rights reserved.

The copyright holder has granted the permission for posting the article here.

\section{Authors}

Renbin Yan, Christy Tremonti, Matthew A. Bershady, David R. Law, David J. Schlegel, Kevin Bundy, Niv Drory, Nicholas MacDonald, Dmitry Bizyaev, Guillermo A. Blanc, Michael R. Blanton, Brian Cherinka, Arthur Eigenbrot, James E. Gunn, Paul Harding, David W. Hogg, José R. Sánchez-Gallego, Sebastian F. Sánchez, David A. Wake, Anne-Marie Weijmans, Ting Xiao, and Kai Zhang 


\title{
SDSS-IV/MaNGA: SPECTROPHOTOMETRIC CALIBRATION TECHNIQUE
}

\author{
Renbin Yan $^{1}$, Christy Tremonti ${ }^{2}$, Matthew A. Bershady ${ }^{2}$, David R. Law ${ }^{3}$, David J. Schlegel ${ }^{4}$, Kevin Bundy ${ }^{5}$, \\ Niv Drory ${ }^{6}$, Nicholas MacDonald ${ }^{7}$, Dmitry Bizyaev ${ }^{8,9}$, Guillermo A. Blanc ${ }^{10,11,12,21}$, Michael R. Blanton ${ }^{13}$, \\ Brian Cherinka ${ }^{14}$, Arthur Eigenbrot ${ }^{2}$, James E. GunN ${ }^{15}$, Paul Harding ${ }^{16}$, David W. Hogg ${ }^{13}$, José R. SÁnchez-Gallego ${ }^{1}$, \\ Sebastian F. Sánchez ${ }^{17}$, David A. Wake ${ }^{2,18}$, Anne-Marie Weijmans ${ }^{19}$, Ting Xiao ${ }^{20}$, and Kai Zhang ${ }^{1}$ \\ ${ }^{1}$ Department of Physics and Astronomy, University of Kentucky, 505 Rose St., Lexington, KY 40506-0057, USA; yanrenbin@uky.edu \\ ${ }^{2}$ Department of Astronomy, University of Winsconsin-Madison, 475 N. Charter Street, Madison, WI 53706-1582, USA \\ ${ }^{3}$ Space Telescope Science Institute, 3700 San Martin Drive, Baltimore, MD 21218, USA \\ ${ }^{4}$ Physics Division, Lawrence Berkeley National Laboratory, Berkeley, CA 94720-8160, USA \\ ${ }^{5}$ Kavli IPMU (WPI), UTIAS, The University of Tokyo, Kashiwa, Chiba 277-8583, Japan \\ ${ }^{6}$ McDonald Observatory, Department of Astronomy, University of Texas at Austin, 1 University Station, Austin, TX 78712-0259, USA \\ ${ }^{7}$ Department of Astronomy, Box 351580, University of Washington, Seattle, WA 98195, USA \\ ${ }^{8}$ Apache Point Observatory, P.O. Box 59, sunspot, NM 88349, USA \\ ${ }^{9}$ Sternberg Astronomical Institute, Moscow State University, Universitetskij pr. 13, Moscow, Russia \\ ${ }^{10}$ Departamento de Astronomía, Universidad de Chile, Camino el Observatorio 1515, Las Condes, Santiago, Chile \\ ${ }^{11}$ Centro de Astrofísica y Tecnologǵas Afines (CATA), Camino del Observatorio 1515, Las Condes, Santiago, Chile \\ ${ }_{12}$ Observatories of the Carnegie Institution for Science, 813 Santa Barbara St, Pasadena, CA, 91101, USA \\ ${ }^{13}$ Center for Cosmology and Particle Physics, Department of Physics, New York University, 4 Washington Place, New York, NY 10003, USA \\ ${ }^{14}$ Dunlap Institute for Astronomy and Astrophysics, University of Toronto, 50 St. George Street, Toronto, Ontario M5S 3H4, Canada \\ ${ }_{16}^{15}$ Department of Astrophysical Sciences, Princeton University, Princeton, NJ 08544, USA \\ ${ }^{16}$ Department of Astronomy, Case Western Reserve University, Cleveland, OH 44106, USA \\ ${ }^{17}$ Instituto de Astronomia, Universidad Nacional Autonoma de Mexico, A.P. 70-264, 04510 Mexico D.F., Mexico \\ ${ }_{18}$ Department of Physical Sciences, The Open University, Milton Keynes, MK7 6AA, UK \\ ${ }^{19}$ School of Physics and Astronomy, University of St Andrews, North Haugh, St Andrews KY16 9SS, UK \\ ${ }^{20}$ Shanghai Astronomical Observatory, Nandan Road 80, Shanghai 200030, China \\ Received 2015 August 17; accepted 2015 November 3; published 2015 December 21
}

\begin{abstract}
Mapping Nearby Galaxies at Apache Point Observatory (MaNGA), one of three core programs in the Sloan Digital Sky Survey-IV, is an integral-field spectroscopic survey of roughly 10,000 nearby galaxies. It employs dithered observations using 17 hexagonal bundles of $2^{\prime \prime}$ fibers to obtain resolved spectroscopy over a wide wavelength range of 3600-10300 $\AA$. To map the internal variations within each galaxy, we need to perform accurate spectral surface photometry, which is to calibrate the specific intensity at every spatial location sampled by each individual aperture element of the integral field unit. The calibration must correct only for the flux loss due to atmospheric throughput and the instrument response, but not for losses due to the finite geometry of the fiber aperture. This requires the use of standard star measurements to strictly separate these two flux loss factors (throughput versus geometry), a difficult challenge with standard single-fiber spectroscopy techniques due to various practical limitations. Therefore, we developed a technique for spectral surface photometry using multiple small fiberbundles targeting standard stars simultaneously with galaxy observations. We discuss the principles of our approach and how they compare to previous efforts, and we demonstrate the precision and accuracy achieved. MaNGA's relative calibration between the wavelengths of $\mathrm{H} \alpha$ and $\mathrm{H} \beta$ has an rms of $1.7 \%$, while that between [N II] $\lambda 6583$ and [O II] $\lambda 3727$ has an rms of $4.7 \%$. Using extinction-corrected star formation rates and gas-phase metallicities as an illustration, this level of precision guarantees that flux calibration errors will be sub-dominant when estimating these quantities. The absolute calibration is better than $5 \%$ for more than $89 \%$ of MaNGA's wavelength range.
\end{abstract}

Key words: atmospheric effects - methods: observational - surveys - techniques: imaging spectroscopy

\section{INTRODUCTION}

Spectrophotometry refers to the calibration of the observed flux density as a function of wavelength to the intrinsic flux density of the target. This calibration is critically important for deriving accurate quantities for many physical properties from spectroscopic measurements of galaxies, including emission line measures of star formation rates (SFRs) and gas-phase metallicities and stellar population parameters from spectral fitting. The success of Sloan Digital Sky Survey (York et al. 2000) would not be possible without its accurate spectrophotometric calibration. In SDSS-I, -II and -III, multiple standard stars were observed simultaneously with the science

\footnotetext{
${ }^{21}$ Visiting Astronomer.
}

targets, and the achieved calibration accuracy is on the order of 5\% (Adelman-McCarthy et al. 2008; Dawson et al. 2013).

The Mapping Nearby Galaxies at Apache Point Observatory (MaNGA) project (Bundy et al. 2015) is an integral field spectroscopic (IFS) survey of nearby galaxies using the $2.5 \mathrm{~m}$ Sloan Foundation Telescope (Gunn et al. 2006) and the BOSS spectrographs (Smee et al. 2013). It is one of three surveys that comprise Sloan Digital Sky Survey-IV (SDSS-IV), which started in 2014 July. With 17 hexagonal fiber bundles (Drory et al. 2015), deployed across each $3^{\circ}$ diameter pointing, MaNGA will obtain spatially resolved spectroscopy for roughly 10,000 nearby galaxies by 2020 . The fiber bundles are made with $2^{\prime \prime}$ fibers and have sizes ranging from $12^{\prime \prime}$ to $32^{\prime \prime}$ diameter in the long axis. The spatial fill factor is $56 \%$. The 
two BOSS spectrographs, each with a blue and a red camera, provide a wavelength coverage from 3600 to $10300 \AA$ at a resolution of $R \sim 2000$.

Different from other previous and current SDSS surveys that target each source with only one fiber, MaNGA will cover and map individual galaxies. This important difference reshapes the goal of spectrophotometry in the IFS context. For MaNGA, we wish to calibrate spectral surface photometry as we explain below.

In spectroscopic studies of external galaxies, stars have always been used as calibrators for spectrophotometry. However, stars are effectively point sources, while external galaxies often appear as extended sources and in the MaNGA sample cannot be approximated as point sources. Because of this difference between the calibrator and the object of study, the detailed approach of spectrophotometry varies depending on the particulars of the instrument and observation setup, and the desired goal of the calibration.

When the spectroscopic aperture is much larger than the size of the point-spread function (PSF) at all relevant wavelengths, flux calibration using a star can be a trivial exercise. When the aperture is smaller or comparable to the size of the PSF, some fraction of the light from a point source will fall outside the aperture and be lost, with the amount of loss depending on the location of the source within the aperture. Usually, instrument apertures are more closely matched to the PSF for the sake of maximizing the obtained signal-to-noise ratio $(\mathrm{S} / \mathrm{N})$ and optimizing spectral resolution. However, apertures placed on an extended source will not see the same amount of flux loss as for point sources for the simple reason that as some light is shifted out of the aperture other light may be shifted in. The exact amount of light either lost or gained in this manner as the effective location of the aperture changes will be a complicated function of the 2D surface brightness profile of the target. In such cases, there are at least three different spectrophotometry goals as applied to galaxy targets.

[A.] Calibrate to the slit- or fiber-aperture flux density $\left(f_{\lambda}\right)$ of a PSF-convolved spatial profile, or in other words, the specific intensity (a.k.a. surface brightness) integrated within the measurement aperture of a PSF-convolved spatial profile. Here the PSF includes the combined effects of atmospheric seeing, the PSF of the telescope and instrument, and chromatic aberration in the whole system. The goal is to correct for the atmospheric attenuation of the flux density and the instrument response, but not to deconvolve the PSF or correct for geometric shifts due to differential atmospheric refraction (DAR).

[B.] Calibrate to the total flux density incident on the atmosphere if the galaxy were a point source. This is in practice straightforward because the same flux correction vector is applied to both stars and galaxies. But it assumes the target galaxies experience the same DAR and aperture flux losses as the stars do, which is usually not true.

[C.] Calibrate to the total flux density derived from imaging photometry assuming that the relative shape of the spectral energy distribution is uniform within the galaxy. The uniformity assumption is appropriate only for certain science cases.

We consider the first of the above options the most fundamental goal for spectrophotometry. It truly reflects what is being measured. It makes no assumption about the property of the extended source to be observed. The only correction required is the system throughput, without any flux correction due to geometric factors. However, this goal is difficult to achieve given practical limitations, especially for single-fiber spectroscopy, as we will detail below. For slit spectroscopy, one approach is to place a slit much wider than the PSF on standard stars to obtain the needed correction, with the caveat that the resulting spectral resolution will be different.

Given the difficulty of actually achieving Goal A, many observational projects have chosen to fall back to Goal B or C. For single-fiber spectroscopy of galaxies, especially distant ones where galaxies are marginally resolved, these can be sufficient for the purpose of deriving redshifts and measuring approximately global spectral properties.

However, in the IFS context, the ultimate goal is to study the internal variations within a galaxy. Therefore, Goal A is the only sensible choice for spatially mapping the specific intensity as a function of wavelength. There are a number of practical difficulties, however, which we discuss in detail in this paper. For MaNGA, we have developed and tested a method to achieve this goal. The approach we present here is broadly applicable to other IFS studies of extended sources.

This paper is organized in the following way. In Section 2, we first discuss the causes of flux loss and error, how spectrophotometry was done in previous generations of SDSS, and the different spectrophotometry needs for integral field spectroscopy. In Section 3, we discuss how we set the requirements for spectrophotometry given the MaNGA science requirements. We then describe our calibration method and the implementation in Section 4, present the resulting spectrophotometry accuracy achieved in Section 5 and summarize in Section 6.

\section{WHAT TO CORRECT: SOURCES OF FLUX ERRORS}

\subsection{Sources of Flux Loss and Flux Error}

To evaluate whether a spectrophotometric calibration method will achieve the above Goal A, we first have to understand the various reasons why observed spectra differ from the intrinsic spectra of the targets. We put these flux losses and erorrs into two categories.

\subsubsection{Throughput Loss}

The first is flux loss due to imperfect throughput of the system, including atmospheric transparency, reflectance and transmission of all optical elements in the telescope and instrument (including fibers), and CCD quantum efficiency. All these throughput losses are a function of wavelength.

\subsubsection{Aperture-induced Flux Error}

The second kind of flux error is due to aperture miscentering which can also lead to wavelength-dependent flux errors. We refer to this as flux error rather than flux loss because for extended sources unaccounted flux can be both added or lost. The list of causes of this kind of flux error differs for point sources and extended sources. Common to both are mechanical alignment errors from manufacturing, guiding errors at the guiding wavelength, and DAR. In detail, the exact source of these errors and their significance depend on the performance of the observing system hardware and the observing strategy. Below, for the specific case of SDSS, we go through each source in detail. 
1. Fiber Positioning: In SDSS, fibers are positioned on science targets by being plugged into custom-drilled aluminum plates that are mounted at the telescope's focal plane. The holes on the plug plates have positional errors from drilling. The fibers are held within their indiviual metal housings (so called ferrules), which are plugged into the holes. The fiber is not always perfectly centered within the ferrule due to limited precision in manufacturing. The plate hole needs to be slightly larger than the ferrule in order for it to be pluggable, and as a result the ferrule will not be perfectly centered within the hole either. The fiber centration error within the ferrule, the hole-ferrule clearance, and the positional error from drilling can stack up to 0 ". $36 \mathrm{rms}$ positional error on the target (see Drory et al. 2015 for the detailed error stack up), as compared to the $2^{\prime \prime}$ diameter fibers used in SDSSIII and IV. The dominant component is the drilling error. A large part of the drilling offset can be measured postdrilling, and in principle could be taken into account in the spectrophotometric calibration. In practice, this was not done in previous generations of SDSS as it was not deemed scientifically essential.

2. Monochromatic Atmospheric Field Distortions: The monochromatic component of the atmospheric refraction (AR) distorts the field in a non-circularly symmetric way when the telescope is not pointed at zenith. When a plate is drilled, the offsets due to AR at the guide wavelength are taken into account according to the hour angle and altitude at which the plate is planned to be observed. However, observations can last several hours during which the magnitude and direction of the AR will change causing a misalignment between the fiber and the target. Given the Sloan Telescope's wide $3^{\circ}$ diameter field of view, the misalignment can be signficant. By tuning the distance between the primary and the secondary mirror, the scale of the field can be adjusted to partially compensate. However, the quadrupole distortion cannot be corrected (for more details, see Section 4.2 of Law et al. 2015). This means some fibers, depending on their positions on the plate, will be offset from the target even if guiding is perfect. The global guiding error for SDSS is expected to be much smaller than all these effects.

For example, at a zenith distance of $18^{\circ}$ (airmass of $1.05)$, the compression of the $3^{\circ}$ field in the altitude direction is 2.4 . Compensating with the scale change, the residual offset due to the $\mathrm{AR}$ for a target on the plate could be somewhere between $0^{\prime \prime}$ and $0 . " 6$ at the guiding wavelength. The global guiding error is on the order of 0 ". 05 .

3. Differential Atmospheric Refraction: The third contributor to the aperture centering error is the differential atmosphere refraction. This means the images of the targets at blue wavelengths are offset from those at red wavelengths. At an airmass of 1.05, the separation between the monochromatic images at 3600 and $10300 \AA$ is 0 "! 54 . At airmass 1.25 , it is 1 ." 27 . For a point source, this means the flux loss due to a finite fixed aperture is different for different wavelengths (e.g., a point source centered in a fiber at one wavelength may fall near the edge of that fiber at another wavelength). For an extended source, this means the fiber is seeing different parts of the source at different wavelengths.
The spectrum one eventually extracts from an individual fiber contains mixed information from different parts of the galaxy. In slit spectroscopy, one could align the slit with the parallactic angle to capture all the flux. For single-fiber spectroscopy on extended sources with internal variations, we will not be able to correct for DAR to get a spectrum for the same physical aperture at all wavelengths, because we cannot correct for flux that we do not observe and is a priori unknown. This is why we excluded DAR corrections in Goal A above, and why Goal A is the most sensible spectrophotometry goal for extended sources.

In SDSS-I to SDSS-III, the approach of Goal B was adopted for spectrophotometry. Due to the different flux loss experienced by point sources and extended sources, there could be significant wavelength-dependent systematics in the flux calibration for each galaxy, especially when DAR is large. For many science topics this may not matter, but avoiding such systematics becomes critical in the context of IFS.

4. Seeing and Chromatic Aberrations: For point sources, aperture losses arise from two additional factors, both of which lead to wavelength-dependent PSF variation. The first is the wavelength-dependent seeing profile. The second is the chromatic aberration of the system. For example, for the Sloan Telescope, the plate is designed to follow the focal plane shape at $5300 \mathrm{~A}$. The focal planes for other wavelengths are different. The resulting PSF shape as a function of wavelength as seen by fibers at different plate locations can be distorted.

The treatment of these effects for extended sources depends on the spectrophotometry goals. For example, for Goal A, these two factors should be included in the intrinsic source properties for which there should be no corrections. What one observes with fiber spectroscopy is the aperture flux of the surface brightness distribution convolved with the wavelength-dependent PSF. One cannot reliably deconvolve the PSF without knowing the intrinsic intensity distribution within each galaxy. If, on the other hand, one adopts Goal B for practical reasons, then galaxies are assumed to experience the same flux loss due to these two factors as stars do, even though this assumption is in general incorrect.

The first three factors above are all related to alignment. Their combined effects are different for stars and galaxies. For stars, a certain fraction of flux is lost as a function of wavelength and the needed correction factor is usually a slow function of wavelength. There are no high-frequency changes to the spectral shape. For galaxies, the impact is more complicated because alignment errors combined with DAR mean that different parts of the galaxy are sampled at different wavelengths.

Given the sources of flux errors above, it is clear that IFS requires calibration of spectral surface photometry (i.e., Goal A), which necessitates corrections only for the throughput loss of the system but not any aperture-induced flux error. However, because we use stars as calibrators, they do experience aperture-induced flux error as well. Thus, to separate these two sources of flux errors for calibration stars, we have to know exactly how the stars are positioned relative to the spectroscopic aperture and the shape of the PSF. 


\subsection{Calibration for Single-fiber Spectrsocopy in SDSS-III/BOSS}

Below we describe the flux calibration method used in SDSS-III/BOSS, since MaNGA is using the same spectrographs and the same fiber size as BOSS. In SDSS-III/BOSS, 20 single fibers per plate were placed on standard stars. They were observed simultaneously with all the science targets. The light from the standard stars experienced the same throughput loss as the science fibers, with a small dependence on airmass. However, every fiber has a different aperture-induced flux error, due to their slightly different alignment error from manufacturing, drilling, and guiding, which are also compounded with DAR.

The observed standard star spectra are first continuumnormalized using a running median filter with a width of 99 pixels $(\sim 110 \AA$ in the blue camera and $\sim 140 \AA$ in the red camera) and then compared with a grid of continuumnormalized Kurucz stellar models with different surface temperature, metallicity $([\mathrm{Fe} / \mathrm{H}])$, and surface gravity to find the best fitting models to all standards on the plate. For each standard, a version of the chosen model which has not been continuum-normalized is reddened using the extinction map of Schlegel et al. (1998) and the extinction law of O'Donnell (1994) and then scaled to match the $r$-band PSF magnitude of the star from its SDSS imaging photometry. The calibration pipeline then compares the observed spectra of each plate's standard stars with the reddened and normalized model spectra to determine a set of correction vectors. These corrections account for both the throughput loss and the aperture-induced flux errors experienced by point sources. Applying these corrections to a galaxy is basically treating galaxies like point sources, what we refer to as Goal B spectrophotometry in Section 1.

Different alignment errors yield different aperture-induced flux errors among a plate's standard stars, leading to correction vectors with significant differences in their overall shapes. First, the low-order shape difference is taken out by dividing each correction vector by a cubic polynomial fit to their ratio to the mean correction vector. Then all these low-order flattened correction vectors for all stars are combined together to derive an "average" wavelength-dependent and airmass-dependent correction vector. Then the pipeline chooses a "best" exposure and corrects the spectra from all the other exposures to match those in the best exposure on an object-by-object basis. This step is required before all exposures can be coadded and involves only low-order polynomial scaling as a function of wavelength. Hence it can remove low-order flux differences caused by different DAR and guiding effects between multiple exposures.

Finally, after all exposures are combined, the pipeline solves for a flux distortion factor to correct for any remaining flux error by comparing synthesized magnitudes from spectra with PSF magnitudes for stars and PSF-equivalent magnitudes for galaxies. Using all galaxy and star targets, the code solves for a low order function that depends on wavelength and plate position for each spectrograph. If the drilling error, guiding error, and DAR can all be approximated by low order functions of wavelength and/or plate position, this step should correct for those errors. On average, Goal B would be achieved although results for individual galaxies could still deviate due to significant fiber mis-alignment.
For Data Releases 6 and 7 (DR6 and DR7) of SDSS-I and -II, the flux calibration method used was the same as that described here. The only difference is that the fibers were $3^{\prime \prime}$ in diameter and standard stars were targeted with 16 fibers per plate. The resulting relative spectrophotometric calibration in SDSS-I and -II has an rms error of 5\% in relative calibration (measured with $g-r$ color) and an rms error of $4 \%$ in absolute calibration ( $r$ magnitude) (Adelman-McCarthy et al. 2008). In SDSS-III/BOSS, with smaller fibers sizes $\left(2^{\prime \prime}\right)$, the error was somewhat worse with an $6.3 \%$ rms error in $g-r$ and $5.8 \% \mathrm{rms}$ error in $r$ for galaxies and stars ${ }^{22}$ (Dawson et al. 2013).

From a practical standpoint, this is nearly the best approach one could take without significantly greater effort given the difficulty to determine exactly how the fibers are positioned relative to each star and each science target. Without that information, it is impossible to separate throughput losses from aperture-induced flux errors. However, for integral field spectroscopy, this challenge must be overcome.

\subsection{Calibration for Integral-field Spectroscopy (SDSS-IV/MaNGA)}

The goal of integral field spectroscopy is to probe the spatially resolved information in an extended source. No assumption about the uniformity of any properties of the target would be appropriate. Each aperture element in an IFS instrument (a fiber in a bundle, or a lenslet in a lenslet array) yields a sampling of the seeing-convolved, aperture-convolved surface brightness profile of the target as a function of wavelength. In calibrating the flux for each aperture element of an integral field unit (IFU), only the throughput loss should be corrected, not any aperture-induced flux errors. Therefore, we will separate these two factors using standard star observations in order to calibrate spectral surface photometry and achieve Goal A.

DAR will still cause each IFU fiber to sample different parts of a target galaxy at different wavelengths. Rather than attempting to correct for this spatial shift, we instead simply compute a position array corresponding to the effective location of each IFU fiber on the sky as a function of wavelength. When the individual fiber spectra are combined together into a rectified data cube (for details see D. R. Law et al. 2015, in preparation) the DAR effect will be removed by reconstructing images of the source at each wavelength using these effective fiber locations. In other words, our goal here is to correct only for non-geometric system throughput losses.

If we could measure all of the light from the calibrators (stars) with large, fully sampled, apertures that delivered the same spectral resolution as our galaxy spectra, then getting the throughput correction would be trivial. This turns out to be difficult in practice. We considered various hardware solutions to separate the throughput loss from the aperture-induced flux error (see Appendix A). Below we first describe what other IFS surveys do for calibration and then present our solution in Section 4.

\subsection{Flux Calibration in other IFS Surveys}

There have been many IFS surveys of galaxies, including SAURON (de Zeeuw et al. 2002), ATLAS3D (Cappellari

\footnotetext{
22 The spectrophotometry error is different for quasar targets in SDSS-III/ BOSS, see Margala et al. (2015).
} 
et al. 2011), DiskMass (Bershady et al. 2010), PINGS (Rosales-Ortega et al. 2010), CALIFA (Sánchez et al. 2012), VENGA (Blanc et al. 2013), and SAMI (Bryant et al. 2014). In all of these surveys except SAMI, galaxies are observed one at a time, and standard stars are observed at different times from the science targets because of instrumental constraints. This practice assumes that the observing conditions are the same between the science exposures and the calibration exposures, which is not always true. This is a major difference from the methodology in MaNGA, in which 17 galaxies are observed simultaneously along with 12 standard stars, enabling independent flux calibration corrections for every exposure. Of particular relevance in motivating our approach are the DiskMass, PINGS, CALIFA, and VENGA surveys, as all of these make use of fiber bundles with incomplete spatial coverage.

The PPak instrument used by DiskMass, PINGS, and CALIFA has 2!.7 fibers $^{23}$, slightly larger than MaNGA, so the aperture loss and DAR effects are smaller. The DiskMass Survey did not do flux calibration as the wavelength coverage was very narrow and the main goal of the survey was to constrain kinematics. In the PINGS survey, the throughput correction (which they call nightly sensitivity function) is derived from the standard star observations by applying a monochromatic aperture correction to the standard star spectrum (Rosales-Ortega et al. 2010). Alignment offset, wavelength-dependent seeing, and DAR would cause the actual aperture-correction to be wavelength dependent. This was not taken into account. According to Rosales-Ortega et al. (2010), when normalized at $4861 \AA$, the resulting relative calibration has a min-to-max variation of $\pm 15 \%$ at $3700 \AA$ and $\pm 10 \%$ at $6850 \AA$. For CALIFA, the original spectrophotometric calibration procedure adopted was in essence very similar to that adopted by PINGS and also did not include the wavelength-dependent aperture correction for standard stars. Since late 2013 and for CALIFA data released in DR2 (GarcíaBenito et al. 2015), an improved calibration scheme was adopted. It uses a set of elliptical galaxies as the calibrator, rather than using standard stars. Because outer regions of elliptical galaxies have very smooth surface brightness profiles, slight alignment offset and DAR would have much less impact on the shape of the spectra. These elliptical galaxies were previously calibrated to the standard spectrophotometric stars by observing both with the PMAS Lens-Array (LArr). As PMAS LArr has a $100 \%$ fill factor, it does not suffer from wavelength-dependent aperture loss. When compared to SDSS images, CALIFA DR2 data have a $5 \%$ rms calibration error in $g$-band and $6 \%$ in $r$-band. The $g-r$ color has a $3 \%$ rms error relative to SDSS images. CALIFA applies a final absolute calibration by registering to SDSS broadband images. This step can take out any remaining absolute calibration error in one band but will keep the relative calibration error between different wavelengths.

The VENGA survey (Blanc et al. 2013) used the VIRUS-P instrument which has $4^{\prime \prime}$ fibers. Any wavelength dependence of the aperture correction for these "fat" fibers is probably small enough to neglect provided the observations are done at reasonably high altitude. Spectroscopic standards were observed with multiple dither positions. The fluxes in multiple fibers in all dither positions are used to fit a fiber-convolved

\footnotetext{
23 Throughout the paper, the fiber sizes given always refer to the flux-sensitive core of the fibers, not the outer diameter of the buffered fiber.
}

PSF profile. Then a monochromatic aperture correction is derived and used to correct the spectra before comparing it with the standard spectrum. The relative calibration accuracy is estimated to be $\sim 8 \%$. Afterwards, the absolute calibration is obtained by comparing the synthesized images from the data cube with the broadband optical images.

The SAMI survey employs a two-step flux calibration process (Allen et al. 2015; Sharp et al. 2015). First, a primary spectrophotometric standard star is observed during the same night as the galaxy observations (but not simultaneously) to provide a low-order calibration for the wavelength-dependent throughput correction. This is done by fitting a PSF model to the fluxes of multiple fibers in the bundle yielding a wavelength-dependent aperture correction. This is then taken into account in deriving the throughput. Each plate also includes a secondary standard star, observed simultaneously as the galaxy observations, which provides the telluric correction and an absolute wavelength-independent flux scaling. Comparing the resulting stellar spectra of the secondary standard stars to broadband photometry, the relative calibration in $g-r$ color is $4.3 \%$ with a systematic offset of $4.1 \%$. By comparing broadband photometry of target galaxies with those obtained from the datacube, the absolute calibration is found to have a systematic offset of $4.4 \%$ and a $1 \sigma$ scatter of $28 \%$. As we detail in later sections, the method we adopted for MaNGA is similar to SAMI, but we observe multiple standard stars through fiber bundles simultaneously with the science targets and we use the guider images to facilitate the PSF fitting.

MaNGA also has much wider wavelength coverage than all of the above surveys. Therefore, the DAR and wavelengthdependent aperture correction have a more significant impact on the MaNGA data.

\section{SCIENCE REQUIREMENTS FOR SPECTROPHOTOMETRY}

The required accuracy of spectrophotometry is determined by the science requirements of the survey. Those that make use of emission-lines are most sensitive to relative spectrophotometry. One goal is to measure the gas phase metallicity in star-forming galaxies, which requires the measurement of multiple emission lines including at least [O $\mathrm{II}] \lambda 3727, \mathrm{H} \beta$, [O III] $\lambda \lambda 4959,5007, \mathrm{H} \alpha$, and [N II] $\lambda \lambda 6548,6583$. They are spread across nearly $3000 \AA$ in wavelength. Thus relative spectrophotometry is crucial. Dust extinction corrections are also needed in computing some of the indicators that involve widely separated lines, such as $\left[\mathrm{N}_{\mathrm{II}}\right] /[\mathrm{O}$ II $]$. Extinction estimates are derived from the Balmer decrement, $\mathrm{H} \alpha / \mathrm{H} \beta$, which is also sensitive to relative spectrophotometric calibration.

For MaNGA, we require that the uncertainty on spectrophotometric calibration does not dominate the uncertainties on the derived SFR and gas metallicities.

\subsection{Calibration Requirement on $\mathrm{H \alpha}$ and $\mathrm{H} \beta$}

First, we describe how the error on spectrophotometry could translate to the error on extinction and SFR.

Below, we use $C_{\lambda}$ to denote the flux calibration vector which needs to be multiplied with the raw flux to get the calibrated flux. For line fluxes, we use $F_{r}(\mathrm{H} \alpha)$ to denote the raw fluxes measured before applying the spectrophotometric calibration, $F_{o}(\mathrm{H} \alpha)$ to denote the flux after calibration, and $F_{c}(\mathrm{H} \alpha)$ to 
denote the flux after extinction correction. ${ }^{24}$ Given these definitions, we have

$$
F_{o}(\mathrm{H} \alpha)=F_{r}(\mathrm{H} \alpha) C_{\mathrm{H} \alpha} .
$$

The uncertainty on $F_{o}(\mathrm{H} \alpha)$ should follow

$$
\left(\frac{\sigma_{F_{o}(\mathrm{H} \alpha)}}{F_{o}(\mathrm{H} \alpha)}\right)^{2}=\left(\frac{\sigma_{F_{r}(\mathrm{H} \alpha)}}{F_{r}(\mathrm{H} \alpha)}\right)^{2}+\left(\frac{\sigma_{\mathrm{H}_{\mathrm{H}}}}{C_{\mathrm{H} \alpha}}\right)^{2} .
$$

We define $c_{1}=C_{\mathrm{H} \alpha} / C_{\mathrm{H} \beta}$, the relative calibration between $\mathrm{H} \alpha$ and $\mathrm{H} \beta$. Extinction is usually derived using the Balmer decrement. We define $r=F_{o}(\mathrm{H} \alpha) / F_{o}(\mathrm{H} \beta)$ and let $\sigma_{r}$ denote the uncertainty of $r$ such that

$$
\begin{gathered}
r=\frac{F_{o}(\mathrm{H} \alpha)}{F_{o}(\mathrm{H} \beta)}=\frac{F_{r}(\mathrm{H} \alpha)}{F_{r}(\mathrm{H} \beta)} c_{1} \\
\left(\frac{\sigma_{r}}{r}\right)^{2}=\left(\frac{\sigma_{F_{r}(\mathrm{H} \alpha)}}{F_{r}(\mathrm{H} \alpha)}\right)^{2}+\left(\frac{\sigma_{F_{r}(\mathrm{H} \beta)}}{F_{r}(\mathrm{H} \beta)}\right)^{2}+\left(\frac{\sigma_{c_{1}}}{c_{1}}\right)^{2} .
\end{gathered}
$$

Taking the Case B Balmer decrement of 2.863 at $T=10^{4} \mathrm{~K}$ and $n=10^{2} \mathrm{~cm}^{-3}$ (Osterbrock \& Ferland 2006), we have

$$
E(B-V)=\frac{2.5}{\left(k_{\mathrm{H} \beta}-k_{\mathrm{H} \alpha}\right)} \log \frac{r}{2.863} .
$$

Here $k_{\mathrm{H} \alpha}$ indicates the total-to-selective extinction for $\mathrm{H} \alpha$. The uncertainty on $E(B-V)$ is

$$
\sigma_{E(B-V)}^{2}=\left(\frac{2.5}{(\ln 10)\left(k_{\mathrm{H} \beta}-k_{\mathrm{H} \alpha}\right)}\right)^{2}\left(\frac{\sigma_{r}}{r}\right)^{2} .
$$

The extinction-corrected $\mathrm{H} \alpha$ flux is

$$
\begin{aligned}
F_{c}(\mathrm{H} \alpha) & =F_{o}(\mathrm{H} \alpha) 10^{0.4 A_{\mathrm{H} \alpha}} \\
& =F_{o}(\mathrm{H} \alpha) 10^{0.4 k_{\mathrm{H} \alpha} E(B-V)} .
\end{aligned}
$$

The uncertainty on $F_{c}$ can be derived as

$$
\left(\frac{\sigma_{F_{c}(\mathrm{H} \alpha)}}{F_{c}(\mathrm{H} \alpha)}\right)^{2}=\left(\frac{\sigma_{F_{o}(\mathrm{H} \alpha)}}{F_{o}(\mathrm{H} \alpha)}\right)^{2}+\left(0.4 k_{\mathrm{H} \alpha} \ln 10 \sigma_{E(B-V)}\right)^{2} .
$$

A common estimate of SFR is derived from the extinction-corrected $\mathrm{H} \alpha$ luminosity $\left(L_{c}(\mathrm{H} \alpha)\right)$. Adopting the SFR calibration given by Kennicutt (1998), $\operatorname{SFR}\left(M_{\odot} \mathrm{yr}^{-1}\right)=$ $7.9 \times 10^{-42} L_{c}(\mathrm{H} \alpha)\left(\mathrm{erg} \mathrm{s}^{-1}\right)$, and assuming zero uncertainty on distances, we have

$$
\left(\frac{\sigma_{\mathrm{SFR}}}{\mathrm{SFR}}\right)^{2}=\left(\frac{\sigma_{L_{c}(\mathrm{H} \alpha)}}{L_{c}(\mathrm{H} \alpha)}\right)^{2}=\left(\frac{\sigma_{F_{c}(\mathrm{H} \alpha)}}{F_{c}(\mathrm{H} \alpha)}\right)^{2} .
$$

Combining Equations (2), (6)-(8), we have

$$
\begin{gathered}
\left(\frac{\sigma_{\mathrm{SFR}}}{\mathrm{SFR}}\right)^{2}=\left(\frac{\sigma_{F_{c}(\mathrm{H} \alpha)}}{F_{c}(\mathrm{H} \alpha)}\right)^{2} \\
=\left(\frac{\sigma_{F_{o}(\mathrm{H} \alpha)}}{F_{o}(\mathrm{H} \alpha)}\right)^{2}+\left(\frac{k_{\mathrm{H} \alpha}}{k_{\mathrm{H} \beta}-k_{\mathrm{H} \alpha}}\right)^{2}\left(\frac{\sigma_{r}}{r}\right)^{2}
\end{gathered}
$$

\footnotetext{
${ }^{24}$ Here, we are referring to extinction intrinsic to the source, not the foreground extinction in the Milky Way.
}

$$
\begin{aligned}
= & \left(\frac{\sigma_{F_{r}(\mathrm{H} \alpha)}}{F_{r}(\mathrm{H} \alpha)}\right)^{2}+\left(\frac{\sigma_{C_{\mathrm{H} \alpha}}}{C_{\mathrm{H} \alpha}}\right)^{2} \\
& +\left(\frac{k_{\mathrm{H} \alpha}}{k_{\mathrm{H} \beta}-k_{\mathrm{H} \alpha}}\right)^{2}\left(\frac{\sigma_{r}}{r}\right)^{2} .
\end{aligned}
$$

Adopting the dust attenuation law of O'Donnell (1994) and $R_{\mathrm{v}}=3.1$, we have $k_{\mathrm{H} \alpha}=2.519$ and $k_{\mathrm{H} \beta}=3.663$. Combining Equations (11) and (4), we have

$$
\begin{aligned}
\left(\frac{\sigma_{\mathrm{SFR}}}{\mathrm{SFR}}\right)^{2}= & 5.85\left(\frac{\sigma_{F_{r}(\mathrm{H} \alpha)}}{F_{r}(\mathrm{H} \alpha)}\right)^{2}+4.85\left(\frac{\sigma_{F_{r}(\mathrm{H} \beta)}}{F_{r}(\mathrm{H} \beta)}\right)^{2} \\
& +4.85\left(\frac{\sigma_{c_{1}}}{c_{1}}\right)^{2}+\left(\frac{\sigma_{C_{\mathrm{H} \alpha}}}{C_{\mathrm{H} \alpha}}\right)^{2} .
\end{aligned}
$$

The right-hand side of the Equation (12) contains four terms. The first two terms are related with the fractional errors of the raw measurements of $\mathrm{H} \alpha$ and $\mathrm{H} \beta$, and the latter two are related with the fractional errors of the relative flux calibration and the absolute calibration. The calibration errors would be uniform across each galaxy, but the errors of $\mathrm{H} \alpha$ and $\mathrm{H} \beta$ would depend on the strength of the lines. One of our science requirements is to measure SFR surface density to 0.2 dex. Flux calibration would not dominate the total error when emission lines are weak, but it would dominate when emission lines are strong. We therefore require that, in regions of strong line detections, the error on SFR estimates due to flux calibration alone needs to be better than 0.05 dex (a fractional error of $11.5 \%$ on SFR estimates). This would ensure that the calibration error be subdominant anywhere $\mathrm{H} \beta$ is not measured to better than $19 \sigma$ (5.2\% fractional error; $\left.0.115^{2}=4.85 \times 0.052^{2}\right)$. We split this error budget ( 0.05 dex on SFR) equally between the relative calibration and the absolute calibration-3rd and 4th term in Equation (12). This means that the relative flux calibration between $\mathrm{H} \alpha$ and $\mathrm{H} \beta$ needs to be measured to better than 3.7\%, and the absolute calibration around $\mathrm{H} \alpha$ needs to be better than $8.1 \%$.

\subsection{Requirement on Relative Calibration between [N II] and $\left[\mathrm{O}_{\text {II }}\right]$}

Similarly, we can derive how gas-phase metallicity measurements are affected by the spectrophotometry error. For example, one important gas phase metallicity indicator is the [N $\mathrm{N}_{\text {II }} \lambda \lambda 6583 /\left[\mathrm{O}_{\text {II }}\right] \lambda 3727$ ratio (Kewley \& Dopita 2002). It is one of the best indicators but requires good spectrophotometric calibration and good extinction corrections. Here we denote the ratio in flux calibration correction between [N II] and $\left[\mathrm{O}\right.$ II] as $c_{2}$, the raw flux measurements as $F_{\text {line }}$, and the extinction-corrected [N $\mathrm{II}] /[\mathrm{O}$ II] ratio as $R$. $R$ can be expressed as

$$
\begin{aligned}
R_{[\mathrm{N} \mathrm{II}] /[\mathrm{O} \mathrm{II}]} & =\frac{F_{[\mathrm{N} \mathrm{II}]} 10^{\left.0.4 A_{[\mathrm{NII}}\right]}}{F_{[\mathrm{O} \mathrm{II}]} 10^{0.4 A_{[\mathrm{OII}}}} c_{2} \\
& =\frac{F_{[\mathrm{N} \mathrm{II}]}}{F_{[\mathrm{O} \mathrm{II}]}} 10^{0.4\left(k_{[\mathrm{N} \mathrm{II}}-k_{[\mathrm{O} \mathrm{III}}\right) E(B-V)} c_{2} .
\end{aligned}
$$


Combining with Equations (4) and (6), the fractional error on $R$ can be written as

$$
\begin{aligned}
\left(\frac{\sigma_{R}}{R}\right)^{2}= & \frac{\sigma_{F_{[\mathrm{NI}]}}^{2}}{F_{[\mathrm{N} \mathrm{II}]}^{2}}+\frac{\sigma_{F_{[\mathrm{OII}]}}^{2}}{F_{[\mathrm{O} \mathrm{II}]}^{2}}+\frac{\sigma_{c_{2}}^{2}}{c_{2}^{2}} \\
& +\left(\frac{k_{[\mathrm{N} \mathrm{II}]}-k_{[\mathrm{O} \mathrm{II}]}}{k_{\mathrm{H} \beta}-k_{\mathrm{H} \alpha}}\right)^{2}\left(\frac{\sigma_{F_{\mathrm{H} \alpha}}^{2}}{F_{\mathrm{H} \alpha}^{2}}+\frac{\sigma_{F_{\mathrm{H} \beta}}^{2}}{F_{\mathrm{H} \beta}^{2}}+\frac{\sigma_{c_{1}}^{2}}{c_{1}^{2}}\right) \\
= & \frac{\sigma_{F_{[\mathrm{N} \mathrm{II}]}}^{2}}{F_{[\mathrm{N} \mathrm{II}]}^{2}}+\frac{\sigma_{F_{[\mathrm{OII}]}}^{2}}{F_{[\mathrm{O} \mathrm{II}]}^{2}}+3.57\left(\frac{\sigma_{F_{\mathrm{H} \alpha}}^{2}}{F_{\mathrm{H} \alpha}^{2}}+\frac{\sigma_{F_{\mathrm{H} \beta}}^{2}}{F_{\mathrm{H} \beta}^{2}}\right) \\
& +\frac{\sigma_{c_{2}}^{2}}{c_{2}^{2}}+3.57 \frac{\sigma_{c_{1}}^{2}}{c_{1}^{2}} .
\end{aligned}
$$

In the above equation, we have adopted the extinction law given by O'Donnell (1994) to compute the coefficient involving the $k$ factors.

According to Kewley \& Dopita (2002), [N II]/[O II] is a good metallicity indicator for regimes where $\log (\mathrm{O} / \mathrm{H})+12$ is greater than 8.6 (approximately $([\mathrm{N} \mathrm{II}] /[\mathrm{O} \mathrm{II}])>-1)$. In this regime,

$$
\begin{aligned}
& \log (\mathrm{O} / \mathrm{H})+12 \\
& =\log \left[1.540+1.266 \log R+0.168 \log ^{2} R\right]+8.93
\end{aligned}
$$

The error on metallicity would be

$$
\begin{aligned}
& \sigma(\log (\mathrm{O} / \mathrm{H})) \\
& =\frac{1}{\ln ^{2} 10} \frac{1.266+2 \times 0.168 \log R}{1.540+1.266 \log R+0.168 \log ^{2} R} \frac{\sigma_{R}}{R} .
\end{aligned}
$$

One of our science requirements is to measure gas phase metallicity $(\mathrm{O} / \mathrm{H})$ to 0.1 dex. Fix $\sigma(\log (\mathrm{O} / \mathrm{H}))$ to 0.1 , we can derive the allowed maximum fractional error on $R$ as a function of $R$. The smaller $R$ is, the tigher the constraint is. At $R=0.1$ (corresponding to $\log (\mathrm{O} / \mathrm{H})+12=8.57$ ), the fractional error on $R$ needs to be smaller than $25.2 \%$ to meet the requirement. Again, the flux calibration will only dominate the error when emission lines are strongly detected. We require that, in regions of strong line detections, the fractional error on $R$ due to flux calibration alone to be less than $10 \%$, which would translate to a maximum error of 0.04 dex on $\mathrm{O} / \mathrm{H}$. There are two terms in Equation (14) that are related with flux calibration. Splitting the error budget equally between these two terms, we result at the requirements on the fractional error of $c_{1}$ and $c_{2}: c_{1}$ needs to be measured to better than $3.7 \%$, and $c_{2}$ needs to be measured to better than $7 \%$. These ensure that the flux calibration error be subdominant until $\mathrm{H} \beta$ is measured to better than $19 \sigma$.

\subsection{Requirement on Uniformity of Calibration among Exposures}

In addition, we also have a requirement on the uniformity of the flux calibration from exposure to exposure. MaNGA combines multiple exposures taken at three different dither positions to synthesize a filled data cube. We need the input exposures to have consistent flux calibration. As simulated by Law et al. (2015), if each exposure has a dither-dependent systematic flux calibration error with an rms of 5\%, a $1 \%$ pixelto-pixel error in the reconstructed data cube would result. If the flux calibration errors are uncorrelated with dither position then they will average out across many exposures and not present a strong requirement on flux calibration accuracy.
To summarize, we require the relative flux calibration between $\mathrm{H} \alpha$ and $\mathrm{H} \beta$ to be measured to better than $3.7 \%$, and that between $\left[\mathrm{N}\right.$ II] and $\left[\mathrm{O}_{\mathrm{II}}\right]$ to be measured to better than $7.0 \%$. Given our conservative requirements, even if the calibration accuracy is worse by a factor of 3 , it would still contribute to the error budget on SFR and metallicity measurements as would a $16 \%$ error on $\mathrm{H} \beta$. We also require the systematic calibration difference among exposures to have an rms less than $5 \%$ for the majority of the wavelength range including all the emission lines mentioned here.

\section{FLUX CALIBRATION METHOD IN MaNGA}

\subsection{Mini-bundles}

MaNGA adopts hexagonally packed fiber bundles with $2^{\prime \prime}$ fiber core diameter and 2."5 center-to-center spacing. This yields a fill factor of $56 \%$. To approach critical sampling of the focal plane PSF, we carry out dithered observations and obtain a uniform reconstructed effective PSF in the stacked data cubes. (For discussion of the observing strategy, see Law et al. 2015.) While the fibers do not provide $100 \%$ coverage for any given exposure, following our approach described in Section 2 (Goal A), we do not try to correct for the flux falling into the gaps between fibers, as it is unknown. The knowledge about those missing regions can only be reconstructed through combining dithered observations.

As discussed in Section 2, we need to separate the throughput loss factor from any aperture-induced flux error. We also have to calibrate each exposure individually as the atmospheric transparency can change with time. These require that we either obtain all the flux included in the PSF of observed standard stars or figure out a way to measure the fraction of the PSF sampled by standard star fiber apertures as a function of wavelength.

The original methods used by SDSS-I to -III will not work well for MaNGA because the miscentering of the fiber relative to the stellar calibration source will be unknown due to drilling error, the clearance between ferrule and the plate hole, the uncertainty in the proper motion of the star, and field differential refraction. Therefore, we cannot accurately predict what fraction of the PSF flux is falling into the fiber at each wavelength even though the PSF can be obtained from the guider camera (Section 4.2).

Our solution is to target standard stars with 7-fiber hexagonal bundles with the same fiber size and fill factor as the science bundles. Given the gaps between the fibers, not all the light will be collected. However, the relative flux ratios between the seven fibers allow an accurate determination of the actual position of the star image inside the bundle. Given a priori knowledge about the PSF shape of the star obtained from the guider images and theoretical knowledge about differential atmosphere refraction, we can accurately reconstruct the fraction of light falling into each fiber at each wavelength. This allows us to estimate the aperture loss separately from the throughput loss.

In the final MaNGA instrument configuration, we use 12 of these 7-fiber mini-bundles per cartridge ${ }^{25}$ to target 12 standard stars. They feed two spectrographs with six mini-bundles per

\footnotetext{
25 A cartridge is a large thick disk containing the fiber assembly, the pseduoslits formed by the fibers, and the structure to hold the plate to be observed. Multiple cartridges are prepared each night for efficient observations. Each cartridge is installed with a plate during the day and fibers in that cartridge are plugged into the plate. At night, when changing field of observation, observers remove the previous cartridge on the telescope and install the cartridge containing the next plate. The cartridge changing process takes just several minutes.
} 
spectrograph, which are further grouped into two fiber assemblies with three mini-bundles each. Details of these and how they are organized on the slithead can be found in Drory et al. (2015).

\subsection{Measuring the PSF with the Guider}

The guider system is important in this process as it provides first-order knowledge about the size and shape of the PSF. We briefly describe the guider system here. Guiding is achieved using 16 coherent imaging fiber bundles plugged in the same plate as all the science fibers. These coherent fiber bundles are collections of thousands individual fiberoptic strands assembled together so that the relative orientation of the individual strands is maintained throughout the length of the bundle. Most of the guide bundles used here are $450 \mu \mathrm{m}$ in diameter and each contain $\sim 10,000$ picture elements with each element being only a few microns across. This is not to be confused with the large fiber bundles for science, which has 19-127 fibers with the core of each fiber having a diameter of $120 \mu \mathrm{m}$ and manufactured in completely different manners.

These coherent imaging bundles are routed to a guider block on the side of the cartridge and imaged by a guide camera. Among the 16 guide bundles, two of them are 24 " in diameter and they are used for field acquisition. The other 14 guide bundles are $7^{\prime \prime}$ in diameter and are used for guiding. Among these 14,4 are positioned with their surface $400 \mu \mathrm{m}$ above the plate surface, four are positioned $400 \mu \mathrm{m}$ below the plate surface, and six are positioned at the plate surface as are the science fibers. This design helps focus the telescope via a comparison of the PSF obtained with the guide bundles above, at, and below the plate surface. The guide bundles are distributed across the plate and provide an estimate for the scale of the field, since the guider images reveal how the stars are offset from their expected positions. The scale of the field can be adjusted by tuning the distance between the primary mirror and the secondary mirror.

During observations, the guider system determines the optimal axis, rotation, and scale adjustments to the telescope that would minimize the distances of the 14 stars from the image centers of their respective guide bundles (determined by the flat image). Under typical seeing conditions, the guider takes an exposure every $27 \mathrm{~s}$ (exposures are $15 \mathrm{~s}$ with $12 \mathrm{~s}$ overhead). There are roughly 33 frames per 15 minute science exposure. We stack the guider images together to obtain an effective PSF for the science exposure. This is a time-average of the varying seeing during the exposure and also includes the effect of guiding uncertainties.

We fit each guide star with a double Gaussian with freely varying amplitudes and widths. We choose double Gaussians to model the PSF because they provide a sufficient approximation to the actual PSF within a diameter of $\sim 4 \times$ FWHM and are very fast to compute. The 8 in-focus guide stars give PSFs that sometimes can vary by as much as 0 ". $1-0$ ". 2 in FWHM. The source of this variation is not completely understood, but a few potential causes have been identified.

First, the curvature of the plate does not conform perfectly to the designed focal plane shape with an error up to a $100 \mu \mathrm{m}$. Given the $f / 5$ focal ratio of the telescope and the plate scale of $60.455 \mu \mathrm{m} \mathrm{arcsec}^{-1}$, focal plane mismatch should contribute at most a 0."33-diameter broadening kernel to the PSF. For 1". 5 seeing (FWHM), the combined PSF should only be broadened by $0 . ! 03$ in FWHM. Therefore, it cannot explain the differences.

Second, the guider output block could have a slight tilt relative to the optical axis of the guider camera. Different guider probes are also not perfectly coplanar to each other, with typical offsets expected to be less than $25 \mu \mathrm{m}$. The guider camera has a much faster focal ratio of $f / 1.4$. Thus, these coplanar offsets are more likely the culprit. If this is the case, it would imply that the PSF seen by the guider and the science IFUs are much more uniform than what the guider camera implies.

Therefore, we pick the sharpest PSF among the six in-focus guide stars as the reference PSF. We denote this PSF profile as $p_{0}(r, \theta)$. In our case, we model this as a circularly symmetric profile so there is no angular dependence but we keep $\theta$ in the formula to indicate it is a $2 \mathrm{D}$ profile. This is modeled from guider images at an effective guiding wavelength of $5400 \AA$.

\subsection{Predicting the Wavelength-dependent PSF}

Next, we need to use the measured PSF at the guide wavelength to predict the wavelength-dependent PSF at the position of the standard star target. There are several factors affecting the PSF at different wavelengths. First, the seeing varies as $\lambda^{-1 / 5}$ (Fried 1966; Boyd 1978). We scale the PSF accordingly depending on its wavelength

$$
p_{\lambda}(r)=p_{0}\left(r\left(\lambda / \lambda_{0}\right)^{1 / 5}\right) .
$$

Here, $\lambda_{0}$ is the guiding wavelength and is equal to $5400 \AA$.

Second, the telescope focal plane changes with wavelength resulting in focus offsets as a function of wavelength and position on the plate. The focal plane shapes as designed are given by Gunn et al. (2006) in Table 5 of that paper. We interpolate to obtain the focus offset at each wavelength according to target position on the plate. The out-of-focus PSF should be computed by convolving the in-focus PSF with a ring kernel as shown in Figure 1, which is the telescope pupil. The outer diameter of the ring is set to $1 / 5$ of the focus offset because the telescope has an $f / 5$ beam. The inner diameter of the ring equals $1 / 10$ of the focus offset, which is set by the size of the secondary mirror. We convert the sizes in length units to angular units using the plate scale of $3.62730 \mathrm{~mm} \mathrm{arcmin}{ }^{-1}$ (Gunn et al. 2006). We denote this kernel $k_{\lambda, d}(r, \theta)$, where $d$ is the distance from the center of the plate.

The PSF we would observe is then

$$
\phi_{\lambda, d}(r, \theta)=p_{\lambda}(r, \theta) * k_{\lambda, d}(r) .
$$

This yields the PSF expected as a function of wavelength and position on the plate.

For computational convenience, we also convolve the above PSF model with the fiber aperture (a $2^{\prime \prime}$ diameter circular step function) to obtain the final profile from which we can simply interpolate to obtain the flux one would get in any $2^{\prime \prime}$ fiber in the 7-fiber mini-bundle.

\subsection{Typing the Star}

The standard stars we select for MaNGA are late-F type main-sequence stars. We require the stars to have no nearby bright neighbors to ensure the wings of the PSF is not contaminated. We select stars with observed magnitudes between 14.5 and 17.2 in the $g$-band. If we cannot find enough stars for a field, we move the faint limit to 17.7 , or 18.2 if 


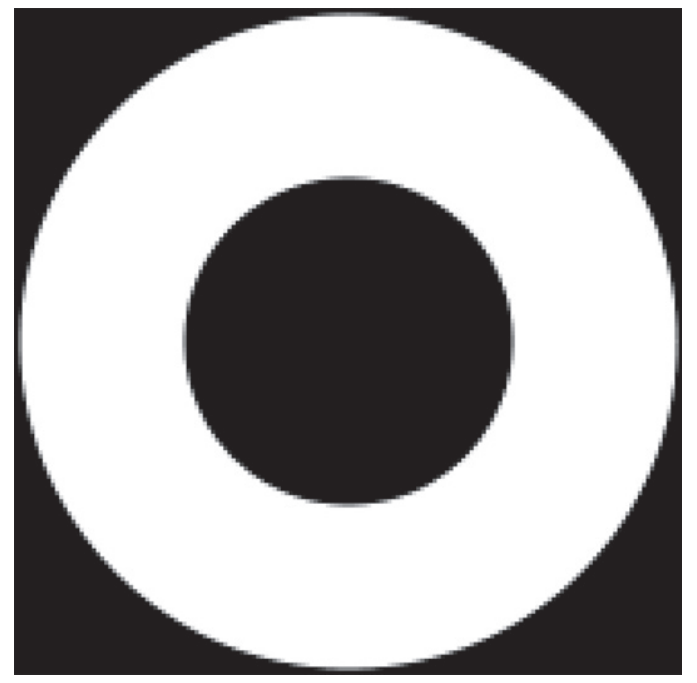

Figure 1. The convolution kernel used in modeling the effect of a focus offset on the PSF.

necessary. Late-F type main-sequence stars have an absolute magnitude ranging roughly between 2.5 and 4 in $g$-band. Our magnitude range ensures that they are at least $1 \mathrm{kpc}$ away. For the MaNGA galaxy program fields, which are all at galactic latitude $(b)$ higher than $20^{\circ}$, these stars are certainly halo stars and are beyond most of the galactic dust.

The reduction pipeline provides a sky-subtracted spectrum for each fiber for each exposure. We first divide these spectra by an initial estimate of the throughput vector, which is the average throughput derived from tens of plates processed by an earlier run of the pipeline. For each mini-bundle, the fiber with the maximum total flux over the whole wavelength range is selected as the reference fiber whose spectrum is used to determine the model spectrum. In fitting the theoretical models, we adopt the same algorithm as used in the SDSS Legacy and SDSS-III/BOSS pipelines, as described in Section 2.2. The resulting model spectra are scaled to match the $r$-band PSF magnitude of the stars.

\subsection{Fitting for the Flux Ratios among Fibers in a Mini-bundle}

To accurately model the aperture loss of a single fiber in the mini-bundle, we first need to know the position of the star relative to the bundle and the PSF. The exact position of the star is uncertain due to uncertainty in astrometry and proper motion, drilling errors, the positional uncertainty of ferrule in its hole, and telescope pointing error. The exact PSF seen by each bundle could also differ from what the best guide star sees due to two reasons: (a) the plate shape is not perfectly matching the focal plane, (b) the smearing of the standard star during the science integration from guiding could be different from the smearing of the guide star, due to the constant scale and rotation adjustments applied by the guider feedback loop which are imperfect. Therefore, we use the flux ratios among the seven central fibers as a function of wavelength to constrain the position of the star and the size of the PSF.

Figure 2 illustrates our method. We choose the fiber with the highest total flux within $3500-10500 \AA$ as the reference fiber. We then sum the flux in eight wide wavelength windows (3500-4000, 4000-4500, 4500-5000, 5000-5500, 5500-6500, 6500-7500, 7500-9000, and 9000-10500 ^) for all fibers and take the ratio between each fiber and the reference fiber for each wavelength window. We run a Markov Chain Monte Carlo with four variables: $x, y$ position of the star, scaling and rotation of the differential atmosphere refraction vector. Given a set of these four parameters, we compute the expected flux ratios from the PSF model. Taking the difference between the observed ratios and the model ratios we compute the $\chi^{2}$ for each step and use the MCMC chain to find the $\chi^{2}$ minimum. The chain often converges within a couple hundred steps. We run it for 500 steps and take the solution giving the minimum $\chi^{2}$. We then scale the PSF to smaller and larger sizes and find the minimum $\chi^{2}$ for each PSF size. Fitting the minimum Chi Square as a function of PSF size by a quadratic function, we find the PSF size that yields the best fit to the flux ratios among fibers, along with the position of the star and DAR. With this best PSF constrained, we rerun the chain for 2000 steps to find the best solution for offsets, rotation and scale. The reason we do not include PSF size as one variable in the MCMC is that the computation of the PSF is a slow process as it involves two convolution procedures.

Throughout this process, whenever summing the observed flux within each wavelength window, we weight each pixel by the inverse variance. The same weighting is applied to the model spectrum. Therefore, the data and the best-fit model should have nearly identical effective wavelength in each wide wavelength window.

\subsection{Deriving the Throughput Loss}

Given the best fit model, we derive the PSF-covering fraction of the fibers, which is defined as the fraction of the flux in a PSF covered by a fiber as a function of wavelength. Figure 3 shows examples of the derived covering fractions for the central fibers in six mini-bundles on one spectrograph for three dithered exposures in a set, and for two airmasses with different levels of AR. We then compute the expected flux of the star by multiplying the theoretical model spectrum with the PSF-covering fraction. Dividing the observed flux by the expected flux from the theoretical model yields the effective correction vector for each star.

The correction vectors derived from the six standard stars on each spectrograph differ slightly in their normalization and the low-order shape. Possible sources of this variation include error in the magnitude of the star, error in the derived covering fractions, error in the model determination, error in the flux extraction from the $2 \mathrm{~d}$ spectra, and the variation of throughput due to airmass differences. The covering fraction error appears to be the dominant source. In some cases, we cannot find a satisfactory fit to all the flux ratios to within the measurement uncertainty. This is probably due to the simplicity of our PSF model and neglect of the guiding error. The true PSF is not circular at all positions on the plate and can be more asymmetric at wavelength extremes. The typical guiding stability of the SDSS Telescope is about 0 ". 12 . Compared to the typical seeing at the site-1."5, most of the time the smearing caused by guiding error should contribute minimally to the final PSF. However, under superb seeing conditions (1"! 0 or better) and at high altitude $\left(>80^{\circ}\right)$ where guiding is worse for this Alt-Az telescope, the guiding error starts to contribute significantly to the integrated PSF over the 15 minute exposure. These factors could contribute to the inconsistency among the correction vectors derived for the standard stars.

Among 54 exposures taken during the commissioning run in 2014 March on four different plates, the average fractional rms 
Plate 7991, Exposure 183338, IFU 59

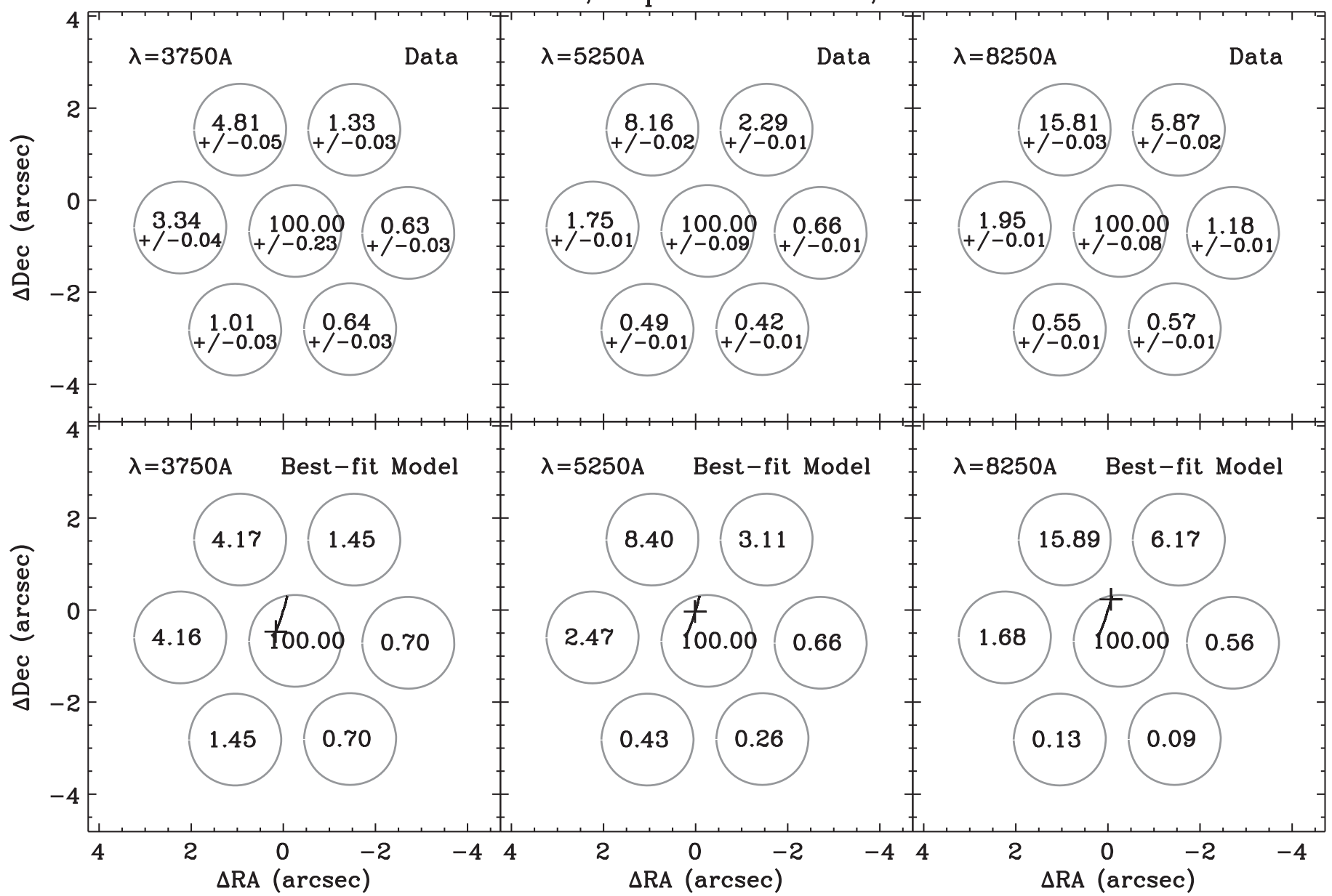

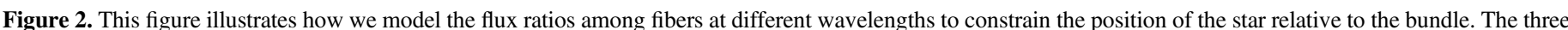

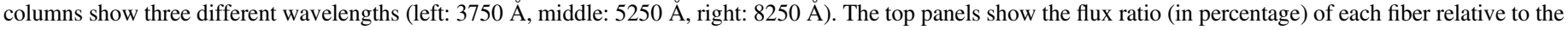

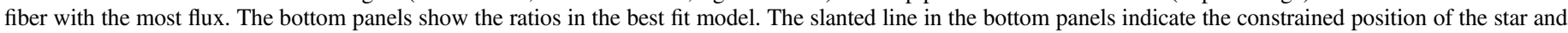

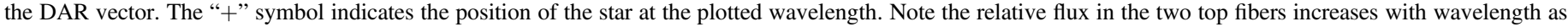

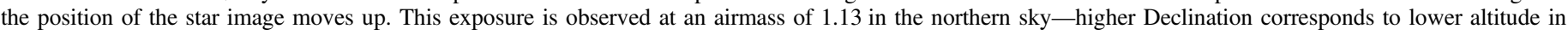
this case.

of the normalization difference among the six stars per spectrograph was $6 \%$, and better than $13.4 \%$ in $95 \%$ of the exposures. The resulting corrections are the mean of all stars and thus have a much smaller uncertainty. Before construting an effective average of the correction vectors, we reject outliers using a series of criteria. Notably, we reject stars that satsify at least one of the following criteria:

1. Having a median $\mathrm{S} / \mathrm{N}$ (among all pixels) lower than $1 / 3$ of the median median-S $/ \mathrm{N}$ of all stars.

2. Having a $\chi^{2}$ from stellar model fit that is more than three times larger than the median $\chi^{2}$ of all stars.

3. Having a $\chi^{2}$ from flux ratio fit that is higher than 100 or the median $\chi^{2}$ of all stars, whichever is larger.

4. We evaluate the median level of each correction vector in two wavelength windows (5300-5350 $\AA$ for the blue camera and 7800-8000 $\AA$ for the red camera). Stars are rejected if their correction vectors are greater than $3 \sigma$ (or $10 \%$, whichever is larger) away from the median levels among all stars in either the blue or the red window. Here, the scatter $(\sigma)$ is computed as the median absolute deviation divided by 0.6745 , which is a robust measurement of scatter for small sample sizes (Beers et al. 1990).
To derive the final correction vector among the vectors of all the stars, we would like to take out the low-order difference among them but keep the high frequency variations in order to keep the constraining power on the high frequency mode. The high frequency variation is due to the telluric absorption by the atmosphere and should be the same among all stars. We would like to use the fact that different stars provides slightly different wavelength sampling. By combining them we can supersample the high frequency variation and provide a more accurate telluric correction. To take out the lower-order difference, we first interpolate all vectors onto a common wavelength grid, and take the average among them. We then divide each vector by the average, and fit the result of this division by a 3rd-order polynomial function. These polynomial functions are a description of the low-order differences among all the correction vectors. We divide each original correction vector (in their original wavelength space) by their respective 3 rd-order polynomial. The resulting vectors now have the same low-order shape but are still in their original wavelength grids, which are slightly different from one another. We call these the low-order-flattened calibration vectors. 


\section{Exposure 177378 Dither S}

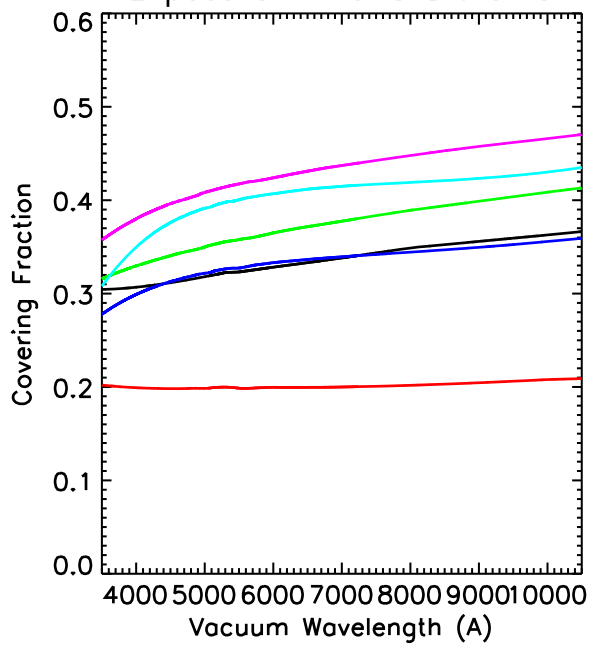

Exposure 181920 Dither $\mathrm{N}$

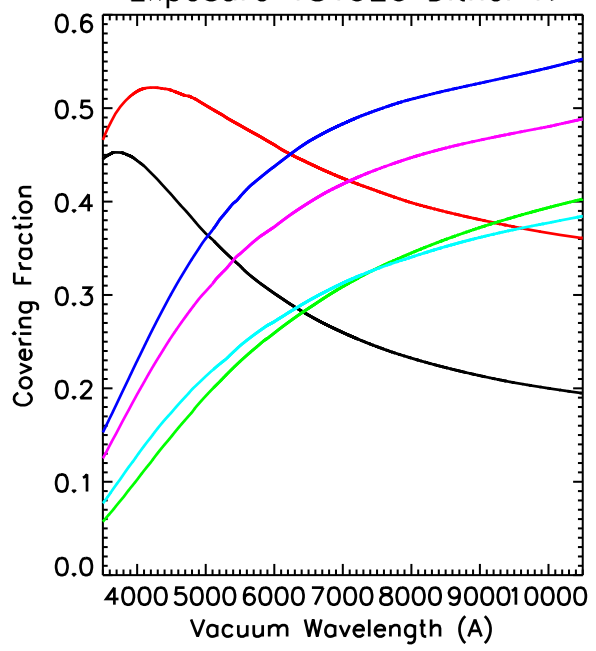

Exposure 177379 Dither E

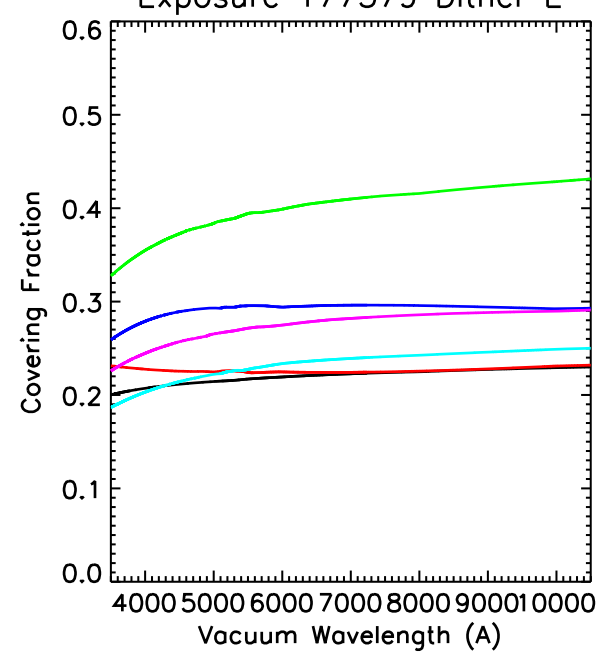

Exposure 181921 Dither $S$

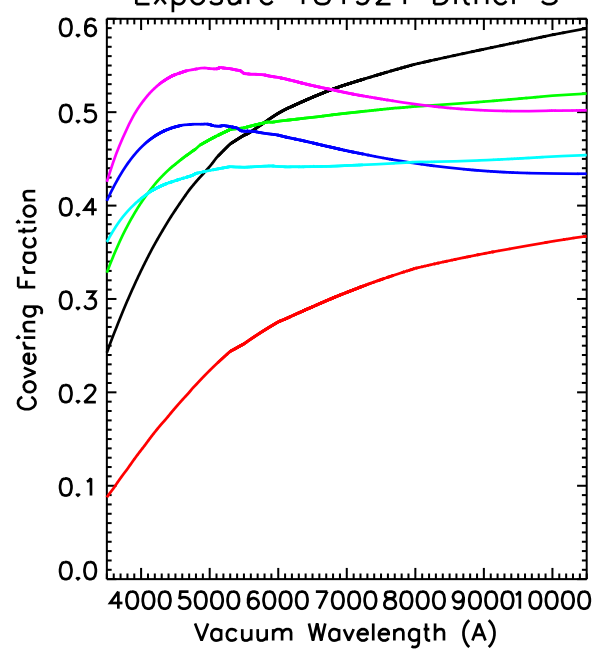

Exposure 177380 Dither N

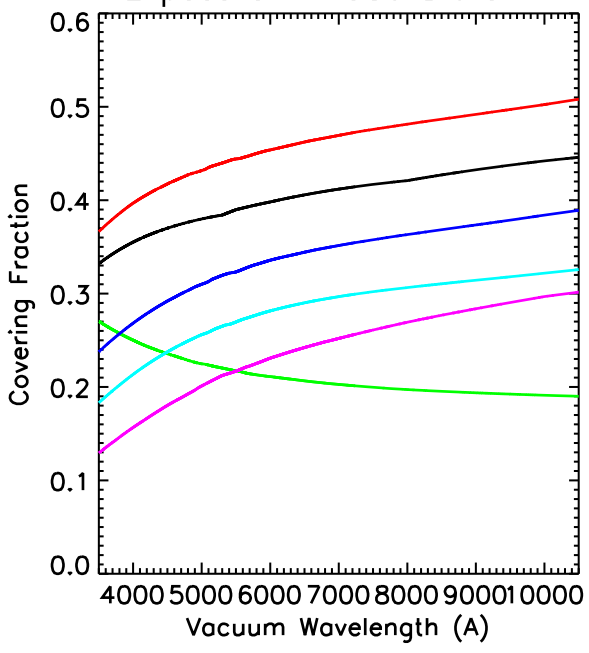

Exposure 181922 Dither E

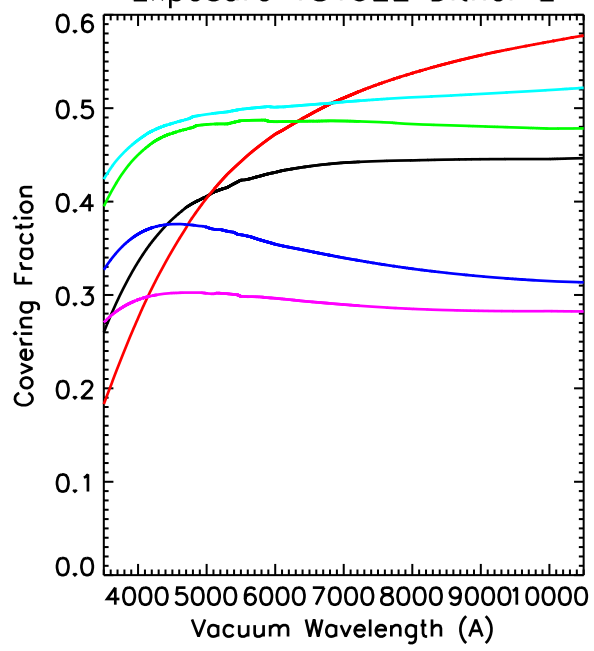

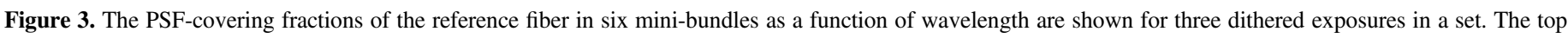

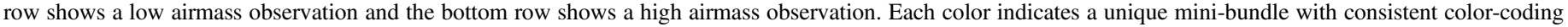
between the two rows. The PSF-covering fraction for the same star can differ signficantly between different airmasses.

The final step is to merge all of these low-order-flattened calibration vectors. With their slightly different wavelength grids, they supersample the spectral resolution element. We fit a b-spline to the merged spectrum with break points separated by $10 n$-pixels in the blue (where $n$ is the number of good stars on a spectrograph) and break points separated by $1.5 n$-pixel in the red. The reason for the higher frequency fit in the red is to be able to sample the telluric absorption lines. Note the pixels here are much smaller than the original pixels because the merging of multiple spectra. The resulting "average" calibration vector is then applied to all of the other spectra in the same spectrograph and from the same exposure. Multiplying this calibration vector with the initial estimate of the throughput yields the final throughput curve of the system including atmospheric transparency. Examples of the derived throughput curves are shown in Figure 4.

These curves can be compared with the throughput curves shown in Figure 38 of Smee et al. (2013), which are defined in the same way. Both the throughput shown here and those of Smee et al. (2013) have made aperture corrections, but in different ways. Smee et al. (2013) made the correction assuming a double Gaussian seeing profile with $1^{\prime \prime}$ FWHM (same for all wavelengths). The observations on which the BOSS throughput was based were conducted under seeing better than 1". 15 and the four standard stars yielding the highest throughput were selected. Our mini-bundles provide much better aperture correction allowing us to derive accurate throughput from observations with much worse seeing. Our throughput is higher than BOSS's by a few percent in the blue and about $5 \%$ in the red. This improvement is consistent with the expectation from our anti-reflection coatings (Drory et al. 2015).

\section{EVALUATING THE CORRECTION ACCURACY}

\subsection{Consistency Among Independent Measurements}

To evaluate the true calibration error as a function of wavelength, we check the consistency in the derived throughput vectors for different exposures, taken at different dither positions, and measured in different spectrographs. This guarantees the throughput vectors we are comparing are completely independent. Different exposures provide different PSF profiles, different dithers provide different sampling of the PSF, and different spectrographs provide different sets of 


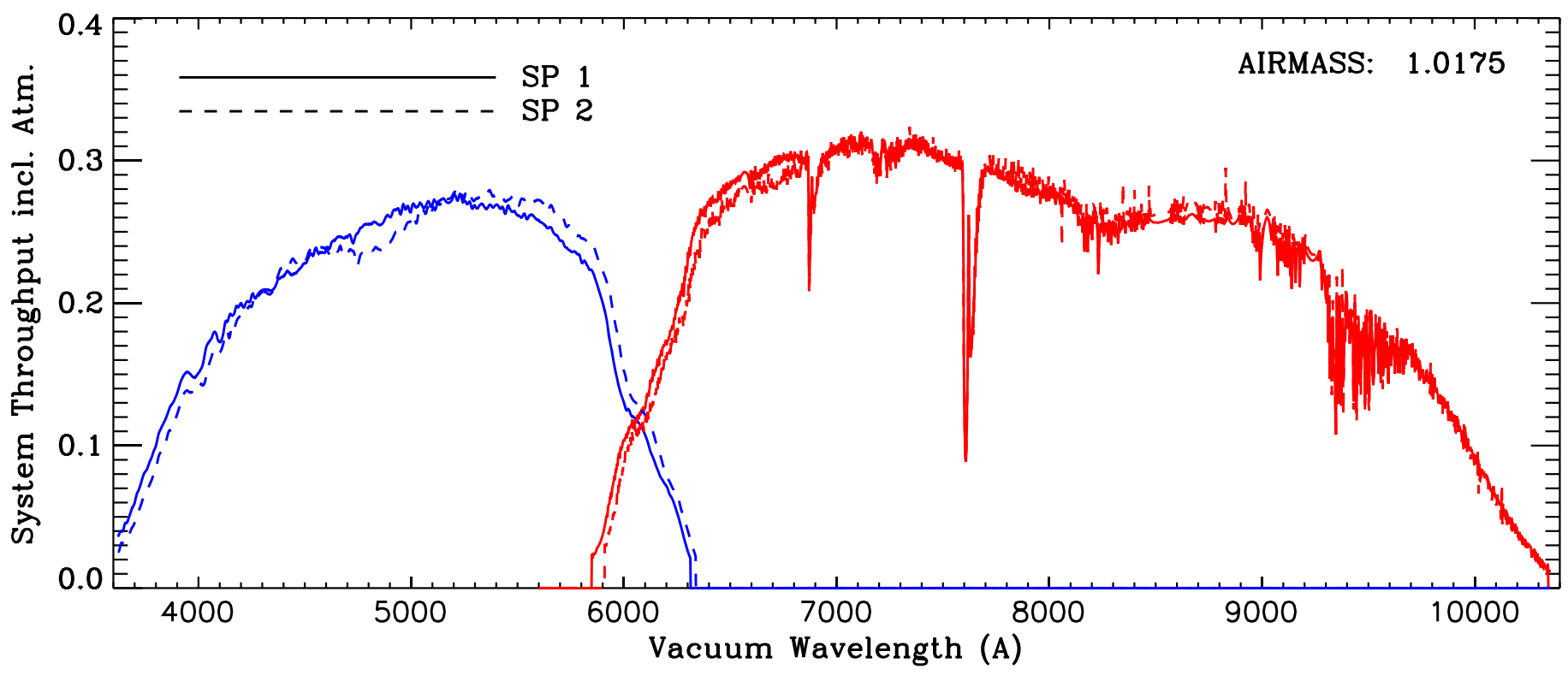

Figure 4. The measured system throughput for one of the exposures (Exposure number: 177380) taken on MJD 56741 at an airmass of 1.0175 . The throughput includes the transparency of the atmosphere, the efficiency of the whole telescope, an average fiber, spectrograph, and the detector. The solid and dashed curves show the throughput for Spectrograph 1 and 2, respectively, with the blue (left) and red (right) colors indicating the two cameras in each spectrograph.

standard stars. There is an intrinsic throughput difference between the two spectrographs but that is fixed for the fixed sets of fiber assemblies (different cartridges have small and negligible differences here). However, because our throughput vector includes the transparency of the atmosphere which varies constantly, the throughput comparison between any two exposures can include an intrinisic difference. To avoid this complication, we only look at pairs of consecutive exposures for which the transparency at the guider wavelength (measured from guider camera images in a broadband filter) differed by less than $3 \%$. This is satisfied by about $80 \%$ of the exposure pairs.

For each such exposure pair, we take the ratio between the throughput vector derived for Spectrograph 1 (SP1) from Exposure 1 and that for Spectrograph 2 (SP2) from Exposure 2. If each individual throughput vector has a fractional error of $x$, the ratio between the two would have a fractional error of $\sqrt{2} x$.

Figure 5 shows the ratio vectors between the throughput vector pairs for 627 such exposure/spectrograph pairs. We always divide SP1 by SP2. The dark lines shows the median ratio at each wavelength, which reflects the intrinsic difference between the two spectrographs. The thinner dark lines show the 2.5-, 15.85-, 84.15-, and 97.5-percentiles of the distribution at each wavelength, corresponding to the enclosed fractions of $1 \sigma$ and $2 \sigma$ limits of a Gaussian distribution. The bottom panel shows the rms of the fractional error divided by $\sqrt{2}$ to show the actual fractional error on each individual calibration. We achieve better than $5 \%$ calibration for $89 \%$ of the wavelength range. This is the random error component of the absolute calibration.

This method also allows us to evaluate the relative calibration accuracy. For each throughput curve, we take the medians in two $20 \AA$ wide windows around $\mathrm{H} \beta$ and $\mathrm{H} \alpha$ (redshifted to MaNGA sample's median redshift). The ratio between the two medians measures the relative calibration $\left(c_{1}\right.$ in Section 3) given by each throughput vector. We then divide the ratio from Spectrograph 1 in Exposure 1 by the ratio from Spectrograph 2 in Exposure 2. The resulting ratio has a fractional rms scatter of $2.4 \%$ among the 627 exposure pairs, corresponding to a $1.7 \%$ fractional error on the relative calibration between $\mathrm{H} \alpha$ and $\mathrm{H} \beta$ for each individual calibration vector. Doing the same calculation for $[\mathrm{N} \mathrm{II}]$ and $[\mathrm{O} \mathrm{II}]$ yields a $4.7 \%$ fractional error on their relative calibration $\left(c_{2}\right)$ for each individual calibration vector. These meet the science requirements specified in Section 3. Given that the distribution of the spectrographs' throughput ratio is fairly close to a Gaussian distribution, these numbers correspond to roughly 68.3percentile of the error distribution.

\subsection{Comparison with Broadband Photometry}

The above comparison provides a measurement of the random component of the calibration error, but it does not determine if there are any systematic offset across all exposures. In this section, we check our absolute accuracy of our spectrophotometric calibration by a comparison to SDSS photometry of galaxies. This comparison is done as part of the MaNGA Data Reduction Pipeline (D. R. Law et al. 2015, in preparation). At a later stage in the pipeline, for each exposure, we register all the spectra taken for each galaxy to the image of that galaxy. Due to the finite mechanical tolerance between the fiber bundles and the holes on the plug plates, and due to imperfect guiding, there is uncertainty in the exact position and rotation of the fiber bundle relative to the galaxy for each exposure. Before we construct the data cube, we need to register the fiber spectra associated with each IFU in each exposure to the imaging. This is done in a manner similar to the method employed by the VENGA Survey (Blanc et al. 2013). First, the synthetic broad-band flux of each fiber is computed by integrating the sky-subtracted, flux-calibrated spectra over the corresponding transmission curve. The code then explores a grid of offsets in position (R.A., decl.) and rotation. At each position on this grid the fiber coordinates are shifted by the corresponding amounts and aperture photometry is performed on a PSF matched SDSS broad-band image using 2!"0 diameter apertures at the corresponding position of each fiber. For 


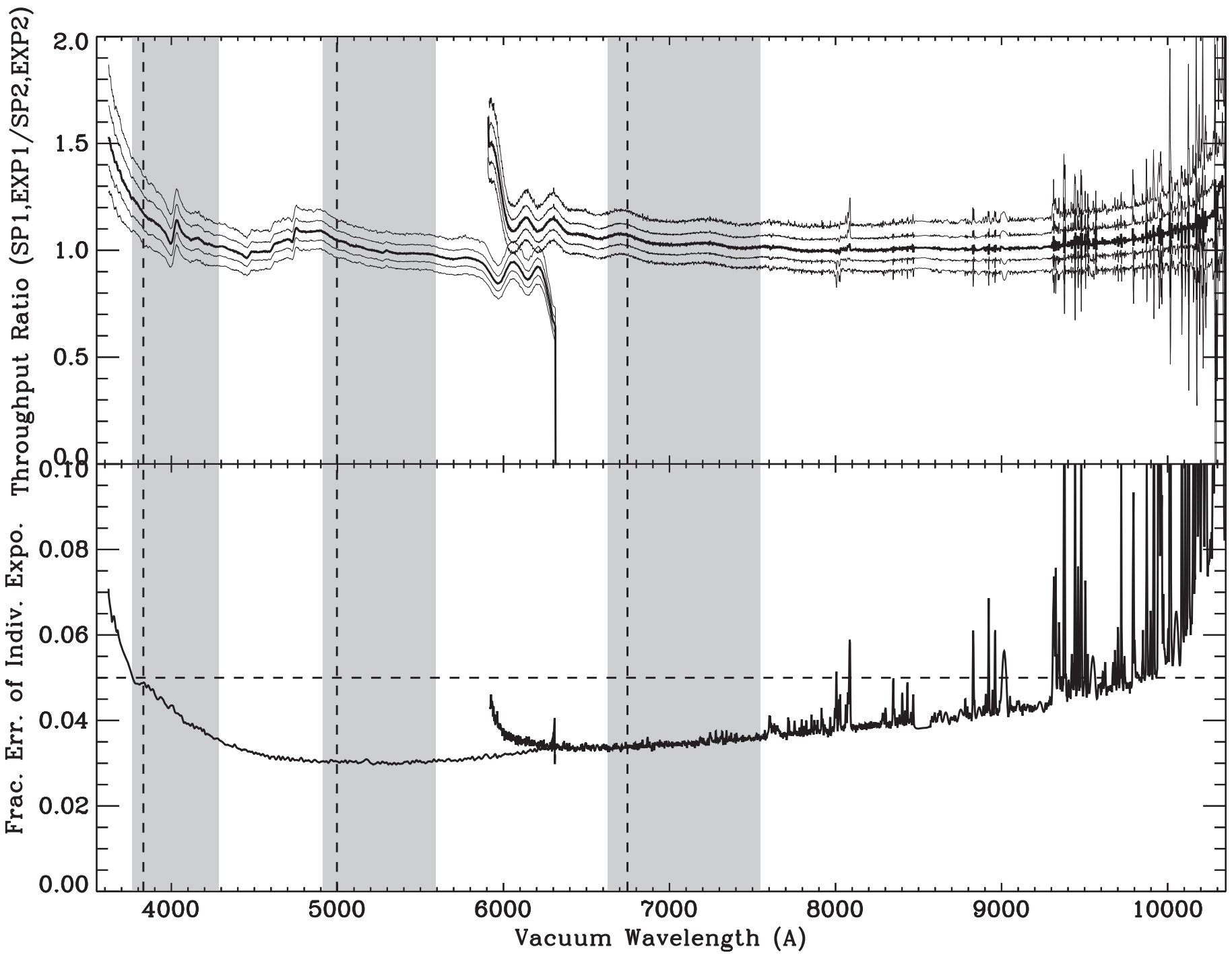

Figure 5. Top: the throughput ratio distribution as a function of wavelength between two completely independent measurements of the throughput curves, constructed by dividing throughput measured in Spectrograph 1 for Exposure 1 and Spectrograph 2 for Exposure 2 for 627 consecutive exposure pairs with different dither positions but similar transparency. The thick line indicates the median ratio among these exposure pairs, which reflects the intrinsic throughput difference between the two spectrographs. The thin lines indicate the 2.5-, 15.85-, 84.15-, and 97.5-percentiles of the distribution at each wavelength, corresponding to the enclosed fractions of $1 \sigma$ and $2 \sigma$ limits of a Gaussian distribution. The curves below (above) $6000 \AA$ are for the blue (red) camera. Bottom: the estimated fractional error of the flux calibration for an individual exposure in a spectrograph. This is derived by taking the standard deviation of the 627 throughput ratio curves, divided by the mean ratio, then divided by $\sqrt{2}$. The horizontal line indicates the 5\% science requirement. For the great majority of the wavelengths, we achieved better than $5 \%$ calibration for the random component (as opposed to the systematic component) of the absolute calibration. The gray bands indicate the positions of [O II] $\lambda 3727, \mathrm{H} \beta$ and $\mathrm{H} \alpha$ ([N II] $\lambda 6583$ is close to $\mathrm{H} \alpha$ ) for the redshift range of the MaNGA sample $(0.01<z<0.15)$. The vertical dashed lines indicate their positions for the median redshift of MaNGA's Primary+sample.

example, for a 61-fiber bundle, there will be 61 synthetic $r$ band flux from MaNGA spectra and $61 r$-band aperture photometry measurements from the image. The code then fits the MANGA synthetic flux of all fibers in a bundle against SDSS broad-band flux using the equation: $F_{\text {SDSS }}=A \times$ $F_{\text {MANGA }}+B$. A perfect flux calibration and sky subtraction in both the spectra and the images would imply $A=1$ and $B=0$. This process yields an evaluation of the flux calibration accuracy for each galaxy in each exposure. Deviation of $A$ from 1 indicate systematics in the absolute flux calibration relative to the imaging. Deviation of $B$ from 0 indicate residuals in sky subtraction in either the imaging or the spectral data. Figure 6 shows the distribution of the flux scaling factors $(A)$ derived for all galaxies on the 64 plates observed before 2015 May 27th, with a total of 753 exposures, and 25,359 IFU-exposure combinations. Occasionally, this astrometry matching fails for reasons unrelated to flux calibration, which result in large $\chi^{2}$. Here, we have removed the $5 \%$ of cases where the $\chi^{2}$ is larger than three indicating bad astrometry matching. The resulting absolute calibration accuracy is better than $4 \%$ in all bands (upper panels in Figure 6) and the relative calibration between bands is better than 3\% (lower panels). This is well within the science requirements for MaNGA. We note that the median value for $A$ is lower than 1 by $2 \%$ for $g$-band and $r$-band, indicating a $2 \%$ systematic difference between SDSS imaging calibration and our spectral data. The error could come from either the imaging or the spectral data, or both. Since we have met our science requirements, we do not try to sort out the source of the systematic difference here and leave it for future investigations. 

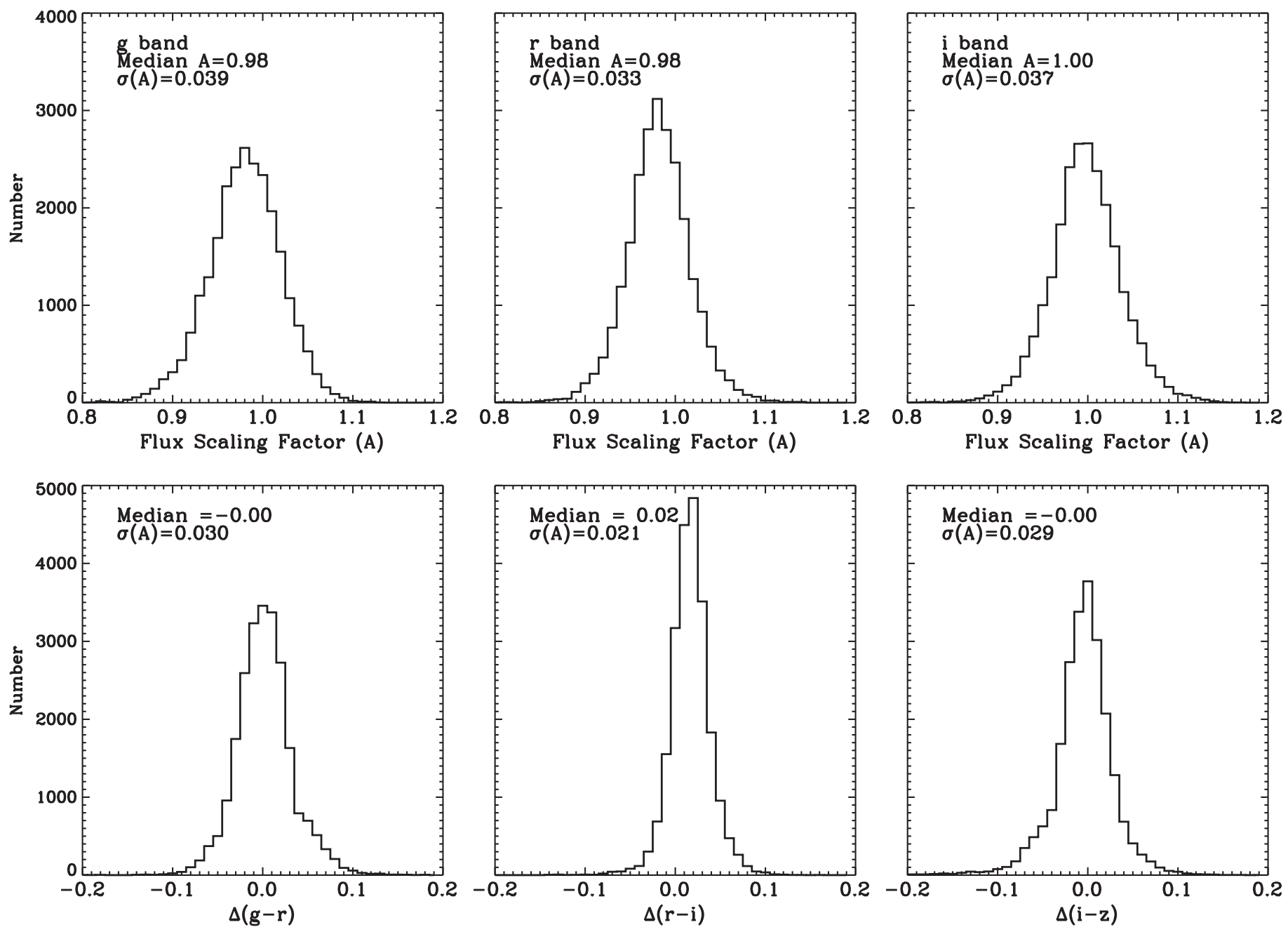

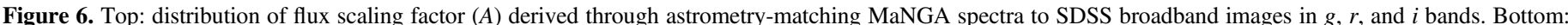
distribution of color residuals implied by the relative flux scaling ratios between different bands.

\section{SUMMARY}

The science goals of integral field spectroscopy require that we strictly calibrate the spectrum of each aperture element in the IFU to the aperture flux density of a PSF-convolved surface brightness profile without artificially accounting for any missing flux due to aperture misalignment or AR. This is a more challenging goal than what is usually required for singlefiber spectroscopy science. We have demonstrated the use of mini-bundles to achieve the separation of the flux loss due to throughput and flux loss due to a finite fiber aperture. The resulting relative calibration uncertainty has an rms fractional error of $1.7 \%$ between $\mathrm{H} \alpha$ and $\mathrm{H} \beta$, and $4.7 \%$ between [ $\mathrm{N}$ II] and [O II]. The absolute calibration is better than $5 \%$ for $89 \%$ of the wavelength range. These meet the science requirements for MaNGA.

There are potential improvements we can make to further increase the accuracy of the spectrophotometry. These could include more detailed modeling of the asymmetric PSF, computing the actual guiding corrections to construct the time-integrated PSF, optimization to the PSF fitting procedure, improvements on the model grid and typing of the star. We could also use a large number of exposures to separate the time-invariant component of the correction vector from the time-dependent component. Since MaNGA science requirements are already met, we leave these ideas for future endeavors.

We thank the referee for the very useful comments which helped improve the paper. A.W. acknowledges support of a Leverhulme Trust Early Career Fellowship. D.B. acknowledges support by grant RSF 14-50-00043.

This project made use of data taken in both SDSS-III and SDSS-IV. Funding for SDSS-III has been provided by the Alfred P. Sloan Foundation, the Participating Institutions, the National Science Foundation, and the U.S. Department of Energy Office of Science. Funding for the Sloan Digital Sky Survey IV has been provided by the Alfred P. Sloan Foundation, the U.S. Department of Energy Office of Science, and the Participating Institutions. SDSS-IV acknowledges support and resources from the Center for High-Performance Computing at the University of Utah. The SDSS web site is www.sdss.org.

SDSS is managed by the Astrophysical Research Consortium for the Participating Institutions in both collaborations. In SDSS-III these include the University of Arizona, the Brazilian Participation Group, Brookhaven National Laboratory, Carnegie Mellon University, University of Florida, the French Participation Group, the German Participation Group, 
Harvard University, the Instituto de Astrofisica de Canarias, the Michigan State/Notre Dame/JINA Participation Group, Johns Hopkins University, Lawrence Berkeley National Laboratory, Max Planck Institute for Astrophysics, Max Planck Institute for Extraterrestrial Physics, New Mexico State University, New York University, Ohio State University, Pennsylvania State University, University of Portsmouth, Princeton University, the Spanish Participation Group, University of Tokyo, University of Utah, Vanderbilt University, University of Virginia, University of Washington, and Yale University.

The Participating Institutions in SDSS-IV include the Brazilian Participation Group, the Carnegie Institution for Science, Carnegie Mellon University, the Chilean Participation Group, the French Participation Group, Harvard-Smithsonian Center for Astrophysics, Instituto de Astrofísica de Canarias, The Johns Hopkins University, Kavli Institute for the Physics and Mathematics of the universe (IPMU)/University of Tokyo, Lawrence Berkeley National Laboratory, Leibniz Institut für Astrophysik Potsdam (AIP), Max-Planck-Institut für Astronomie (MPIA Heidelberg), Max-Planck-Institut für Astrophysik (MPA Garching), Max-Planck-Institut für Extraterrestrische Physik (MPE), National Astronomical Observatory of China, New Mexico State University, New York University, University of Notre Dame, Observatório Nacional/MCTI, The Ohio State University, Pennsylvania State University, Shanghai Astronomical Observatory, United Kingdom Participation Group, Universidad Nacional Autónoma de México, University of Arizona, University of Colorado Boulder, University of Oxford, University of Portsmouth, University of Utah, University of Virginia, University of Washington, University of Wisconsin, Vanderbilt University, and Yale University.

\section{APPENDIX A}

\section{ALTERNATIVE CALIBRATION OPTIONS CONSIDERED}

For MaNGA, we developed and tested several flux calibration methods. In this appendix, we discuss other flux calibration options we considered but did not adopt, as these can potential be useful in other situations.

As discussed in Section 2, we need to separate the throughput loss factor from any aperture-induced flux error. One concept was to use large fibers to get all the light from the star, which would be insensitive to differential atmosphere refraction at modest airmass and would suffer little aperture loss. However, there are practical limitations on the fiber size due to the increasing stiffness of large fibers. Experiments at Washburn Laboratories suggested that $5^{\prime \prime}(300 \mu \mathrm{m})$ was the largest fiber size that would be workable. With moderately good seeing conditions of 1.13 and an airmass of 1.12 , with 0 " 15 miscentering errors, a $5^{\prime \prime}$ fiber loses $<1 \%$ of the PSF flux.

However, given that MaNGA's dither pattern traces an equilateral triangle 1." 44 on a side, if we place the center of a $5^{\prime \prime}$ fiber at the center of the dithering triangle, the light losses at different dither positions can increase to as much as $10 \%$ in the blue. To avoid this $10 \%$ light loss, one has to use three sets of standard stars, with each set designed for a different dither position. Considering the larger footprint of the fibers on the $\mathrm{CCD}$, this would require a much larger allocation of our CCD real estate to calibration sources than our chosen method.

Another problem with this large-fiber scheme is that all of the IFU science fibers have a $2^{\prime \prime}$ core. The $5^{\prime \prime}$ fibers would therefore have a different spectral resolution. As a result, they would not be suitable for correcting high-frequency wavelength variations such as telluric absorption features. To achieve the telluric correction, one would have to use both $5^{\prime \prime}$ and $2^{\prime \prime}$ fibers to target standard stars. Again, the total number of fibers allocated for calibration becomes prohibitive.

To measure the telluric correction at the same spectral resolution, another method we considered was to target standards with $5^{\prime \prime}$ fibers coupled to a single $2^{\prime \prime}$ fiber or a 7-fiber hexagonal bundles of $2^{\prime \prime}$ fibers, then feed the $2^{\prime \prime}$ fibers to the spectrographs. If the standard star light collected by the $5^{\prime \prime}$ fiber always emerged with a uniformly illuminated beam, coupling the output beam to a single fiber or a 7-fiber bundle would yield the necessary flux information without a loss of resolution. However, because the star will not illuminate the $5^{\prime \prime}$ fiber uniformly, given the short fiber length, the light will not be completely homogenized inside the $5^{\prime \prime}$ fiber. This is illustrated in Figures 7 and 8 . The scrambling of light inside a $2 \mathrm{~m}$ long fiber is insufficient to reduce monochromatic flux calibration uncertainties below 3.5\% level even if the output end were measured with a mini-bundle of $72^{\prime \prime}$-fibers. Given the DAR, the resulting calibration would also have a wavelengthdependence at this level. Increasing the fiber length to $25 \mathrm{~m}$ long would reduce the uncertainty to $0.3 \%$. However, the fiber throughput would be reduced significantly in the blue if the fibers are much longer than $2 \mathrm{~m}$ (roughly a $20 \%$ decrease in throughput at $400 \mathrm{~nm}$ going from 2 to $25 \mathrm{~m}$ fiber length). Therefore, MaNGA did not pursue this approach. The solution MaNGA adopted is presented in Section 4.

\section{APPENDIX B COMPARISON WITH A TRADITIONAL SPECTROPHOTOMETRIC STANDARD STAR}

In this appendix, we illustrate our calibration accuracy using a traditional spectrophotometirc standard star observed with our system. One should keep in mind that this is a single data point so it cannot be used to establish the statistics of our calibration accuracy. Nonetheless, it is useful to check. During our commissioning observation, we placed a bundle on the standard star HZ 21 for this purpose.

We would also like to note that producing a spectrum for a star using our setup requires an additional step than producing a calibrated spectrum for a fiber in a bundle. For our galaxy targets, we just need to apply the average throughput correction. For a star, we also need to know the PSF-covering fraction for that specific star. Because the individual PSFcovering fraction as a function of wavelength is more uncertain than the average of many stars, the result could only be worse than the actual flux calibration accuracy we achieve in the galaxy data. Nonetheless, this provides a conservative indication of our absolute calibration error.

We compare our derived HZ 21 spectra with the standard spectrum given by STScI's CALSPEC database ${ }^{26}$, which is derived by combining HST/STIS observations with spectra taken by J. Oke (Oke 1990; Bohlin et al. 2001; Bohlin 2007). Figure 9 (top panel) shows the standard spectrum given by the CALSPEC database and the average spectrum we obtained from six dithered exposures. The two spectra trace each other fairly well. It is worth noting our groundbased spectrum have very good telluric correction that it is as smooth in telluric regions as the $H S T /$ STIS spectrum which is not affected by telluric features. Our spectrum has a much higher spectral

\footnotetext{
${ }^{26}$ http://www.stsci.edu/hst/observatory/crds/calspec.html
} 


\section{$2 \mathrm{~m}$ length of $300 \mu \mathrm{m}$ core FBP}
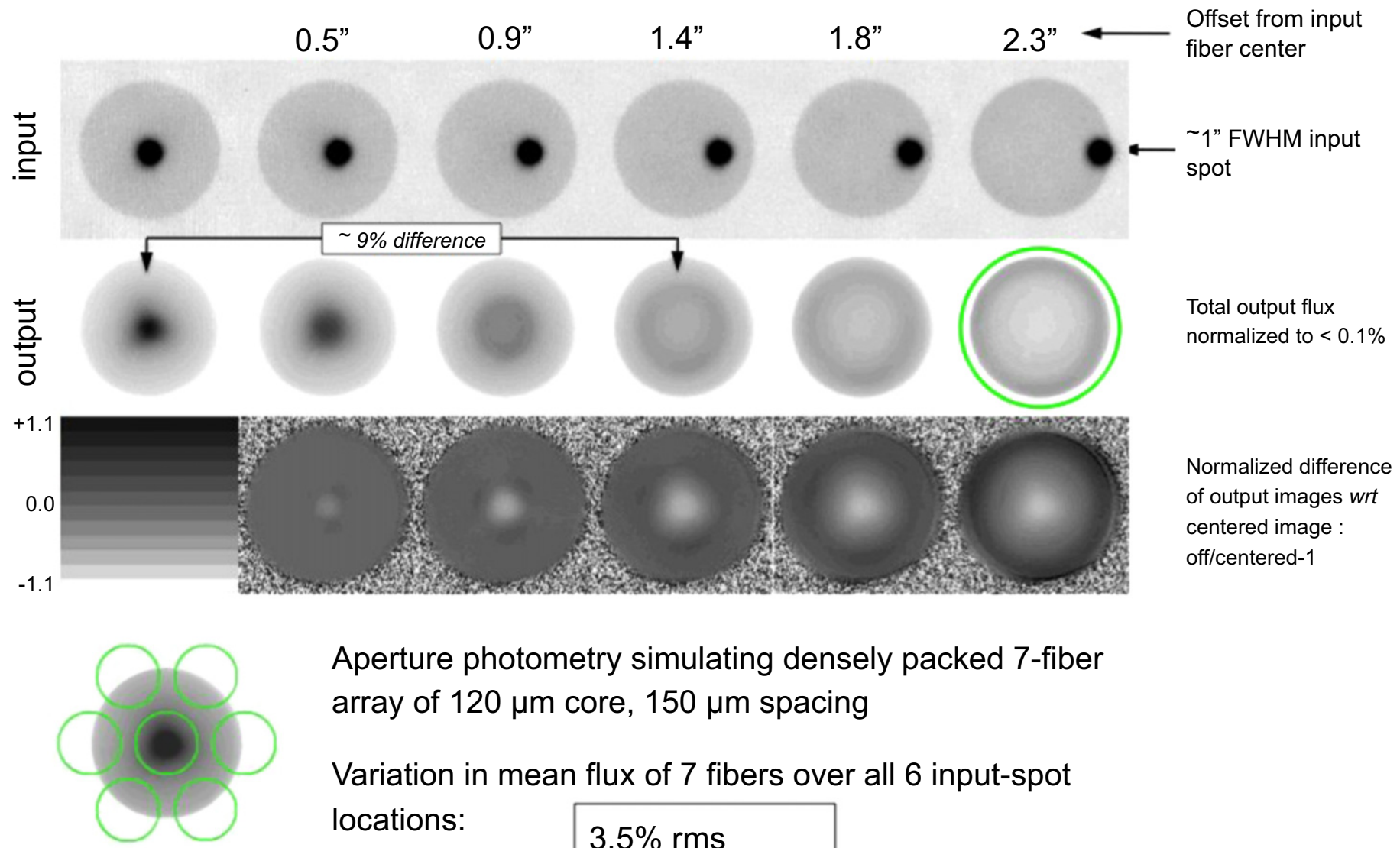

\section{Aperture photometry simulating densely packed 7 -fiber array of $120 \mu \mathrm{m}$ core, $150 \mu \mathrm{m}$ spacing}

\section{locations: \\ $3.5 \% \mathrm{rms}$ \\ $8.9 \%$ full range}

Variation in mean flux of 7 fibers over all 6 input-spot

Figure 7. Result of light scrambling inside a $2 \mathrm{~m}$ long fiber with a $300 \mu \mathrm{m}$ core. The top row shows the six different input beam location relative to the fiber. The middle row shows the output beams. The bottom row shows the normalized difference of output images relative to the centered image. It shows the scrambling of light inside the $2 \mathrm{~m}$ long fiber is insufficient to always feed a smaller fiber with the same fraction of light, as the position of the star changes within the bundle due to dithered observations or DAR. Even if we couple an 7-fiber mini-bundle at the output end, the variation would still be at the several percent level. Additionally, DAR will make the fraction of light recovered by the $2^{\prime \prime}$ fibers a function of wavelength.
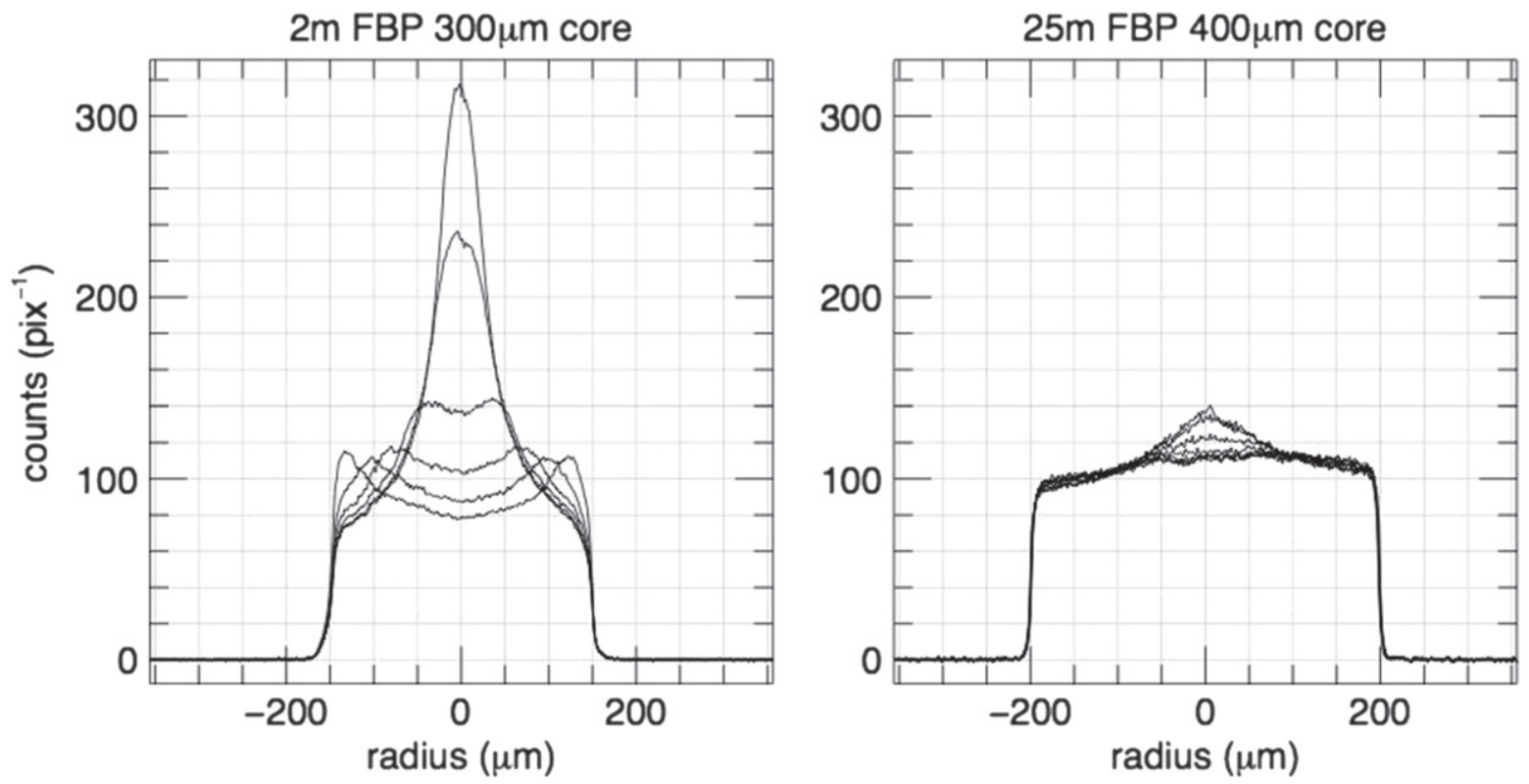

Figure 8. The 1d image profiles of the output beams as presented in Figure 7. The two panels show the results of a $2 \mathrm{~m}$ long fiber with a $300 \mu \mathrm{m}$ core (left) and a $25 \mathrm{~m}$ long fiber with a $400 \mu \mathrm{m}$ core (right). The shorter fiber provides insufficient scrambling. 


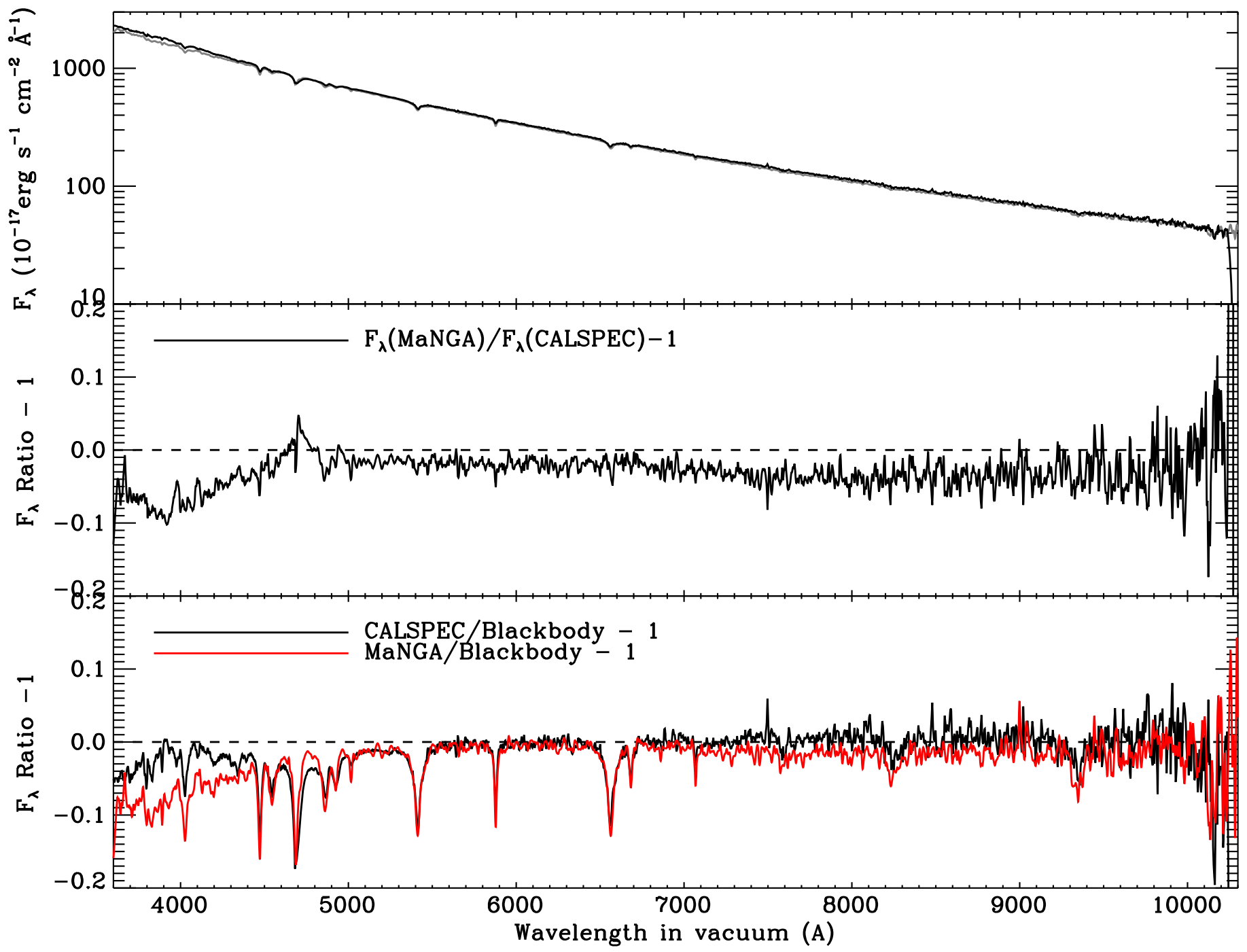

Figure 9. Upper panel: spectra of an Oke standard star, HZ 21, as given by the CALSPEC database (dark black line) and that obtained from the average of six MaNGA exposures (gray line). The systematic error is very small, which is detailed in the bottom panel. Middle panel: fractional deviation of the average derived spectrum for HZ 21 from six exposures, after convolving to the resolution of the CALSPEC spectrum. Bottom panel: fractional deviations of our HZ 21 spectrum and CALSPEC spectrum relative to a blackbody spectrum with $T=100,000 \mathrm{~K}$ normalized around 6000-6100 А. This shows both our spectrum and CALSPEC spectrum have residual systematic errors in the blue wavelengths at a level of 5\%-10\%. Our spectrum is better than CALSPEC between $4600-5000 \AA$ but worse below $4600 \AA$. Note this observation of one star does not fully reflect the statistical accuracy of our flux calibration.

resolution as one could see from the depth of many lines, such as He II $\lambda 4686$. In the middle panel, we convolved our spectra to the resolution as given by CALSPEC, then derived the fractional deviation from the CALSPEC spectrum. The residual show some large scale tilt in certain wavelength windows and some small-scale features. It is quite plausible that there are systematics in the CALSPEC spectrm at this level as well. Since a hot white dwarf's spectrum is very close to a blackbody, we can compare both spectra to a blackbody spectrum to check the systematic error in them. In the literature, there are discrepant measurements for the effective temperature for $\mathrm{HZ} 21$, ranging from around 50,000 K (Koester et al. 1979) to $100,000 \mathrm{~K}$ (Oke \& Shipman 1971; Reynolds et al. 2003). Both our spectrum and CALSPEC spectrum agree much better with a $100,000 \mathrm{~K}$ blackbody spectrum for wavelengths redder than $5000 \AA$. In the bottom panel of Figure 9, we divide both spectra by a $100,000 \mathrm{~K}$ blackbody that has been normalized to each spectrum between $6000-6100 \AA$. Since the blackbody spectrum has no absorption lines, only the line-free regions reflect the residual systematics in the data.

This comparison indicates both our spectrum and the CALSPEC spectrum have some small systematics. CALSPEC spectrum has a very broad dip between 4000 and $5000 \AA$ with a 5\% maximum deviation, and a dip below $3900 \AA$. The part of the CALSPEC spectrum between $4683 \AA$ and $5381 \AA$ is from Oke (1990) and is stitched together with $H S T$ /STIS spectrum at $4683 \AA$ around the line center of He II $\lambda 4686$. Our spectrum shows a tilt blueward of $4600 \AA$ that goes down to $-10 \%$ at $3800 \AA$ and a slight tilt redward of $6800 \AA$ of $1 \%-2 \%$. We suspect these systematics could originate from the error in the derived PSF-covering fractions for the F-star standards on this plate and that for HZ 21, which can be due to the simplified assumptions we make about the PSF regarding its circular symmetry and how it changes with focus offset. Given our reported statistics in Section 5.1, the systematics shown in this single spectrum is generally within $1 \sigma$, and at $\sim 2 \sigma$ at the worst part. 


\section{REFERENCES}

Adelman-McCarthy, J. K., Agüeros, M. A., Allam, S. S., et al. 2008, ApJS, 175,297

Allen, J. T., Croom, S. M., Konstantopoulos, I. S., et al. 2015, MNRAS, 446, 1567

Beers, T. C., Flynn, K., \& Gebhardt, K. 1990, AJ, 100, 32

Bershady, M. A., Verheijen, M. A. W., Swaters, R. A., et al. 2010, ApJ, 716, 198

Blanc, G. A., Weinzirl, T., Song, M., et al. 2013, AJ, 145, 138

Bohlin, R. C. 2007, in ASP Conf. Ser. 364, The Future of Photometric, Spectrophotometric and Polarimetric Standardization, ed. C. Sterken (San Francisco, CA: ASP), 315

Bohlin, R. C., Dickinson, M. E., \& Calzetti, D. 2001, AJ, 122, 2118

Boyd, R. W. 1978, JOSA, 68, 877

Bryant, J. J., Owers, M. S., Robotham, A. S. G., et al. 2014, arXiv:1407.7335

Bundy, K., Bershady, M. A., Law, D. R., et al. 2015, ApJ, 798, 7

Cappellari, M., Emsellem, E., Krajnović, D., et al. 2011, MNRAS, 413, 813

Dawson, K. S., Schlegel, D. J., Ahn, C. P., et al. 2013, AJ, 145, 10

de Zeeuw, P. T., Bureau, M., Emsellem, E., et al. 2002, MNRAS, 329, 513

Drory, N., MacDonald, N., Bershady, M. A., et al. 2015, AJ, 149, 77

Fried, D. L. 1966, JOSA, 56, 1372
García-Benito, R., Zibetti, S., Sánchez, S. F., et al. 2015, A\&A, 576, A135

Gunn, J. E., Siegmund, W. A., Mannery, E. J., et al. 2006, AJ, 131, 2332

Kennicutt, R. C. 1998, ARA\&A, 36, 189

Kewley, L. J., \& Dopita, M. A. 2002, ApJS, 142, 35

Koester, D., Liebert, J., \& Hege, E. K. 1979, A\&A, 71, 163

Law, D. R., Yan, R., Bershady, M. A., et al. 2015, AJ, 150, 19

Margala, D., Kirkby, D., Dawson, K., et al. 2015, arXiv:1506.04790

O’Donnell, J. E. 1994, ApJ, 422, 158

Oke, J. B. 1990, AJ, 99, 1621

Oke, J. B., \& Shipman, H. L. 1971, in IAU Symp. 42, White Dwarfs, ed. W. J. Luyten (Dordrecht: Springer), 67

Osterbrock, D. E., \& Ferland, G. J. 2006, Astrophysics of Gaseous Nebulae and Active Galactic Nuclei (Sausalito, CA: University Science Books)

Reynolds, A. P., de Bruijne, J. H. J., Perryman, M. A. C., Peacock, A., \& Bridge, C. M. 2003, A\&A, 400, 1209

Rosales-Ortega, F. F., Kennicutt, R. C., Sánchez, S. F., et al. 2010, MNRAS, 405,735

Sánchez, S. F., Kennicutt, R. C., Gil de Paz, A., et al. 2012, A\&A, 538, A8

Schlegel, D. J., Finkbeiner, D. P., \& Davis, M. 1998, ApJ, 500, 525

Sharp, R., Allen, J. T., Fogarty, L. M. R., et al. 2015, MNRAS, 446, 1551

Smee, S. A., Gunn, J. E., Uomoto, A., et al. 2013, AJ, 146, 32

York, D. G., Adelman, J., Anderson, J. E., Jr., et al. 2000, AJ, 120, 1579 